九州大学学術情報リポジトリ

Kyushu University Institutional Repository

\title{
ASYMPTOTIC PROPERTIES OF SMOOTHED vs. UNSMOOTHED CONDITIONAL DISTRIBUTION FUNCTION ESTIMATORS
}

Mehra, K. L.

Department of Statistics and Applied Probability, University of Alberta

Krishnaiah, Y. S. Rama

Osmania University

Rao, M. Sudhakara

Osmania University

https://doi.org/10.5109/13424

出版情報: Bulletin of informatics and cybernetics. 25 (1/2), pp.71-97, 1992-03. Research Association of Statistical Sciences

バージョン :

権利関係 : 


\title{
ASYMPTOTIC PROPERTIES OF SMOOTHED vs. UNSMOOTHED CONDITIONAL DISTRIBUTION FUNCTION ESTIMATORS*
}

\author{
By \\ K.L. Mehra, Y.S. Rama Krishnaiah** and M. Sudhakara RaO**

\begin{abstract}
Let $\left\{\left(X_{i}, Y_{i}\right): i=1,2, \ldots\right\}$ be a sequence of independent identically distributed random vectors in $\mathscr{R}^{2}$ with an absolutely continuous distribution, and let $G_{x}(\cdot)$ denote the conditional distribution function of $Y_{1}$ given $X_{1}=x$, assuming that it exists. In this paper, the asymptotic normality and almost sure convergence rates for smoothed rank nearest neighbor and NadarayaWatson type estimators of $G_{x}(\cdot)$ are established. It is also shown, using the concept of deficiency, that smoothed estimators are superior (asymptotically) to the corresponding unsmoothed ones under appropriate choice of the smoothing kernels.
\end{abstract}

\section{Introduction}

Let $\left\{\left(X_{i}, Y_{i}\right), i \geq 1\right\}$ be a sequence of independent identically distributed random vectors with a common continuous distribution function (d.f.) $H$ and marginal d.f.'s $F$ and $G$, respectively. Further, let $G_{x}$ denote the (regular) conditional d.f. of $Y$ given $X=x \in \Lambda(F) \subset \Re=\mathscr{R}^{(1)}=$ real line $(\Lambda(F)=$ support of $F)$, assuming that it exists and, for each $0<\lambda<1$, let $q_{x}(\lambda)=G_{x}^{-1}(\lambda)=\inf \left\{y \in \mathscr{R}^{(1)}: G_{x}(y) \geq \lambda\right\}$ denote the $\lambda$ th conditional quantile of $G_{x}, x \in \Lambda(F)$. While literature is replete with work on the estimation of unconditional (joint and marginal) distribution and the quantile functions $H, F, G$ and $F^{-1}$, work on the estimation of conditional distribution and qunatile functions $G_{x}$ and $G_{x}^{-1}$, respectively, started only a decade or so ago, with the pioneering work of Stone [13] and those of Stute [16], [17] on weak and almost sure (a.s.) convergences of conditional empirical (c.e.) d.f.'s and the related empirical processes. (See also Horvath and Yandell [5] and Härdle et al. [4] for a.s. convergence rates of c.e.d.f.'s and those of Stute [17], Samanta [11] and Mehra et al. [9] on a.s. and weak convergences of conditional quantile estimators.) The above papers dealing with the estimation of conditional functions have a commonality in that they are all based on the kernel method of estimation; however, only the last two deal with "smoothed" estimators.

Department of Statistics and Applied Probability, 434 Central Academic Building. University of Alberta, Edmonton, Canada T6G 2G1

* The above research was supported in part by a CRF-NSERC Grant from the University of Alberta and the Grant No. A-3061 from the National Sciences and Engineering Research Council of Canada

*** On lcave from Osmania University. Hyderabad, India. 
The object of the present paper is to establish the asymptotic normality and a.s. convergence rates for the "smoothed" Rank Nearest Neighbor (RNN) and NadarayaWatson (NW) type estimators of conditional d.f.'s and also study their asymptotic efficiencies and deficiencies relative to the corresponding "unsmoothed" estimators of the conditional d.f. $G_{x}(\cdot)$. The asymptotic properties, including Bahadur Representation. of the corresponding inverse type conditional quantile function estimators are dealt with in Mehra, Rama and Rao [8].

In case the underlying distributions are smooth, it would seem natural to consider smoothed estimators for their estimation. For the unconditional distribution functions, such estimators have been recommended by Efron [2] and Mack [7] among others, and have been shown to be superior by Falk [3] (see also Reiss [10]) using the relative deficiency criterion, provided the smoothing kernel employed satisfies a certain "positivity" property. We shall prove below a similar result for smoothed c.e.d.f"s.

Consider now the s.c.e.d.f. defined by

$$
\begin{aligned}
G_{n x}(y) & =\left(n a_{n n} t_{n}(x)\right)^{-1} \sum_{i=1}^{n} W\left(a_{n}^{-1}\left(F_{n}(x)-F_{n}\left(X_{i}\right)\right), a_{n}^{-1}\left(y-Y_{i}\right) \text { if } t_{n}(x)>0\right. \\
& =0 \text { if } t_{n}(x)=0,-x<y<\infty,
\end{aligned}
$$

where $t_{n}(x)=\left(n a_{n}\right)^{-1} \sum_{i=1}^{\prime \prime} W_{n}^{*}\left(\left(F_{n}(x)-F_{n}\left(X_{i}\right)\right) / a_{n}\right)$ with $W^{*}(\cdot)=W(\cdot, x), W(\cdot, y)$ $=\int_{-\infty}^{y} k(\cdot, v) d v, k$ a suitable bivariate probability kernel, $F_{n}(x)=n^{-1} \sum_{i=1}^{n} I_{\left[X_{i}<x\right]}$ and $\left\{a_{n}\right\}$ a sequence of bandwidths with $a_{n} \downarrow 0$ but $n a_{n} \rightarrow x$, as $n \rightarrow x$. In the sequel, we shall actually consider $\widehat{G}_{n x}$ in a slightly more general form relative to (1.1) (see (3.1) below) where $W\left(\cdot, a_{n}^{-1} v\right)$ is replaced by $W_{n}(\cdot, v)$, with $W_{n}(\cdot$,$) a sequence of suitable$ bivariate kernel functions, possibly of higher order; see Remark 3.5) satisfying the assumptions A.III below. The c.e.d.f. $\widehat{G}_{n x}$ defined by (1.1) is a proper (probability) d.f. and a "smoothed" version of the one considered by Stute [17] (see also Horvath and Yandell [5]), provided the bivariate kernel function $W$ satisfies appropriate smoothness conditions in respect of the second argument. We shall refer to it or its generalization (3.1) as a smoothed RNN estimator of $G_{x}$. Samanta [11] also considered the so called smoothed Nadaraya-Watson version, say $\widehat{G}_{m x}^{*}$ of (1.1) with $x$ and $X_{i}$ in place of $F_{n}(x)$ and $F_{n}\left(X_{i}\right)$ respectively. From our standpoint, however, the estimator $\widehat{G}_{n x x}$ is superior to $\widehat{G}_{n, x}^{*}$. This is because the ratio $\left(\sigma_{0}^{2} / \sigma_{0}^{* 2}\right)$ of asymptotic variances of suitably normalized $\widehat{G}_{n x}$ and $\widehat{G}_{n x}^{*}$ equals the value $f(x)$ of the marginal density $f$ of $X_{1}$ at $x$ (see Theorems 3.1(b) and 3.2(b)) which is usually less than one for most values of $x \in \Lambda(F)$. However, the results of this paper cover both the RNN and the NW type smoothed and unsmoothed estimators of c.d.f.'s. The results obtained in Section 3 are valid, under appropriate conditions, for kernels of first as well as higher orders for the comparison of smoothed us unsmoothed c.d.f. estimators. They are conditioned by the choice of appropriate bandwidth sequences $\left\{a_{n}\right\}$ and the order of the kernel functions employed.

The paper is organized as follows: In Section 2, are given the notation, assumptions and some preliminaries. Section 3 contains the weak convergence and a.s. convergence rates of estimators of conditional d.f. $G_{r}(\cdot)$. The asymptotic relative efficiencies and deficiencies of smoothed vs. unsmoothed estimators are studied in Section 4. The final 
section contains some concluding remarks.

\section{Assumptions and Preliminaries}

In this section, we shall state all assumptions that are needed in the sequel and refer to them as need arises in proving our results below. These assumptions hold for a variety of bivariate kernels including, for example, the commonly employed product kernels in literature (cf. (4.3) below and Samanta [11]). We shall also state, for convenience, certain well known results that are needed for the proofs. The $C_{i}$ 's and $c_{i}$ 's that appear in the proofs denote generic constants.

\subsection{Assumptions}

A.I.(i) The joint d.f $H$ is continuous.

(ii) $H$ is absolutely continuous with bounded density $h$ and marginal d.f's $F$ and $G$ with bounded densities $f$ and $g$ respectively.

(iii) $H(u, v)$ and $F(u)$ have continuous bounded (partial) derivatives upto $(m+$ $1)^{\text {th }}$ order for an integer $m \geq 1$ and at all $v,-\infty<v<\infty$, and all $u$ in a $n g h d$ $N_{x}$ of $x \in \Lambda(F)$.

A.II. For fixed $x \in \Lambda(F)$ and $v \in \mathscr{R}$ the conditional d.f. $G_{\eta}^{*}(v)=G\left(v \mid F^{-1}(\eta)\right)$ possesses $(m+1)$ continuous partial derivatives in $\eta, 0<\eta<1$ in a $n g h d N^{*}$ of $\lambda=F(x)$ with

$$
\sup _{\eta \in N^{*}} \sup _{v \in \mathcal{R}}\left|\frac{\partial^{m+1}}{\partial \eta^{m+1}}\left(G_{\eta}^{*}(v)\right)\right|<x .
$$

A.III. The sequence $\left\{W_{n}(t, s):-\infty<t, s<x, n \geq 1\right\}$ of bivariate kernel functions satisfies

(i) for each fixed $s \in \Re$ and $n=1,2, \ldots, W_{n}(t, s)$ is a bounded twice differentiable kernel function in $t \in \mathscr{R}$ that vanishes outside $[-1,1]$;

(ii) for each fixed $t \in \mathscr{R}$ and $n=1,2, \ldots, W_{n}(t, s)$ is bounded and of bounded variation in the second argument and satisfies $\lim _{s \rightarrow-\infty} W_{n}(t, s)=0$ and $\lim _{s \rightarrow \infty}$ $W_{n}(t, s)=W_{n}^{*}(t)$ for some function $W_{n}^{*}$ on $R$.

(iii) for each fixed $n=1,2, \ldots$, some positive integer $m$ and each $t$ and $s \in \mathscr{R}$, respectively $\int s^{i} d W_{n}(t, s)=0$ and $\int t^{i} W_{n}(t, s) d t=0$ for $i=1, \ldots, m$ with $\int|s|^{m+1} d\left|W_{n}(t, s)\right|$ and $\int|t|^{m+1} d\left|W_{n}(t, s)\right|$ finite $((m, m)$ is said to be the order of the kernel $W_{n}$ which can also be $\left(m_{1}, m_{2}\right)$ with $m_{1} \neq m_{2}$; see Remark 3.3 below);

(iv) for some real sequence $\left\{b_{n}\right\}$ satisfying $a_{n}=O\left(b_{n}\right)$ and $b_{n}^{m+1}=O\left(n^{-\frac{1}{2}} a_{n}^{-\frac{1}{2}}\right.$ $\left.\left(\log a_{n}^{-1}\right)^{\frac{1}{2}}\right), \quad \int_{|s| \geq b_{n}}|s|^{m+1} d m_{n}^{*}(s)=O\left(b_{n}^{m+1}\right)$ as $n \rightarrow \infty$, where $m_{n}^{*}(s)=$ $\int W_{l l}(t, s) d t$

(v) for each fixed $t \in[-1,1]$ and $j=0,1,2, V_{n l}^{(j, 0)}(t, s)=\left[W_{n}^{(j, 0)}(t, s) / W_{l l}^{*}(j)(t)\right] \rightarrow$ $I_{[s \geq 0]}$ and $W_{n}^{*(j)}(t)=W_{n}^{(j, 0)}(t, x) \rightarrow k_{l}^{(j)}(t)$, as $n \rightarrow x$, where $k_{1}$ is a univariate twice continuously differentiable bounded kernel function vanishing outside $[-1,1]$. 
A.IV. Let $\left\{a_{n}\right\}$ be a bandwidth sequence such that $a_{n} \downarrow 0$ and as $n \rightarrow \infty$,

(i) $n a_{n} \rightarrow x$ and $\left(\log a_{n}^{-1} / \log \log n\right) \rightarrow x$;

(ii) $n a_{n}^{r} \rightarrow \infty$ for some $3 \leq r<5$ and $n a_{n}^{r^{* *}}=O$ (1) for some $r^{*}>r$, as $n \rightarrow x$;

(iii) $\left(n a_{n}\right)^{1 / 2} b_{n}^{(m+1)} \rightarrow 0$, as $n \rightarrow \infty$, where $b_{n}$ is as given in A.III(iv).

\subsection{Preliminaries}

We now state, as Proposition 2.1, a few well-known results available in literature which are needed to establish the results of this paper: Let $H_{n}(x, y)=n^{-1} \sum_{i=1}^{n}$ $I_{\left[X_{i} \leq x, Y_{i} \leq y\right]}$ denote the empirical d.f. of $\left\{\left(X_{i}, Y_{i}\right): 1 \leq i \leq n\right\}$ and $F_{n}$ and $G_{n}$ the corresponding marginal e.d.f.'s of $\left\{X_{i}: 1 \leq i \leq n\right\}$ and $\left\{Y_{i}: 1 \leq i \leq n\right\}$, respectively. Let $\left\{\beta_{n}(t): 0 \leq t \leq 1\right\}$ denote the empirical process defined by $\beta_{n}(t)=U_{n}\left(F^{-1}(t)\right), 0 \leq t$ $\leq 1$, where $U_{n}(x)=n^{\frac{1}{2}}\left[F_{n}(x)-F(x)\right], x \in \Re$. Further, let

$$
w_{n}(\delta)=\sup _{|t-s| \leq \delta}\left|\beta_{n}(t)-\beta_{n}(s)\right|
$$

denote the oscillation modulus of the process $\left\{\beta_{n}\right\}$. Then we have (see Lemma 2.4 and Theorem 2.14 of Stute [14] and Kiefer [6])

Proposition 2.1. Under the assumptions A.I(i) and A.IV(i),

$$
\begin{gathered}
\lim _{n \rightarrow \infty}\left\{w_{n}\left(a_{n}\right) /\left(2 a_{n} \log a_{n}^{-1}\right)^{\frac{1}{2}}\right\}=1 \text { a.s. ; } \\
\left\{w_{n}\left(a_{n}\right) / a_{n}^{\frac{1}{2}}\right\}=o_{p}(1), \text { as } n \rightarrow \infty \text {, and }
\end{gathered}
$$

(iii) $\sup \left\{n^{\frac{1}{2}}\left|H_{n}(x, y)-H(x, y)\right|:(x, y) \in \mathscr{R}^{(2)}\right\}=O\left((\log \log n)^{\frac{1}{2}}\right)$, a.s., as $n \rightarrow x$.

\section{Asymptotics of $G_{n \cdot x}$}

In this section, we shall establish the asymptotic normality and a.s. convergence rates for $G_{n x x}$ defined by (1.1). We first note that for each fixed value of the first argument $\left\{W\left(\cdot, v a_{n}^{-1}\right) / W^{*}(\cdot)\right\}$ is a sequence of functions of bounded variation converging weakly to the d.f. function with unit mass at zero. (Note that $\underset{v \rightarrow \infty}{\sum_{x}}\left\{W\left(\cdot, a_{n}^{-1} v\right)\right\}$ $\left.W^{*}(\cdot)\right\}=1, \underset{v \rightarrow-x}{\stackrel{L}{\rightarrow}}\left\{W\left(\cdot, a_{n}^{-1} v\right) / W^{*}(\cdot)\right\}=0$.) Such a sequence has been termed as "Heaviside" sequence by Walter and Blum [18]. Also note that $G_{n x}$ is a convolution type estimator of $G_{x}$ and as such, as remarked by Mack (1984), has a definite advantage over the non-convolution type estimators (for example, those based on the trigonometric series method, etc). The latter type, despite their finite sample global "optimality", are frequently in implicit form and, consequently, are quite intractable from the statistical analysis standpoint.

As stated above, we shall henceforth in this section deal with $G_{n, x}$ in a slightly more general form given by

$$
G_{n x}(y)=\left(n a_{n}\right)^{-1}\left(t_{n}(x)\right)^{-1} \sum_{i=1}^{n} W_{n}\left(\left(F_{n}(x)-F_{n}\left(X_{i}\right)\right) / a_{n}, y-Y_{i}\right)
$$




$$
=a_{n}^{-1}\left(t_{n}(x)\right)^{-1} \iint W_{n}\left(\left(F_{n}(x)-F_{n}(u)\right) / a_{n}, y-v\right) d H_{n}(u, v),
$$

where $t_{n l}$ is as defined in (1.1) with $W^{*}(\cdot)$ replaced by $W_{n}^{*}(\cdot)=W_{n}(\cdot, x)$ and the bivariate function $W_{l l}$ satisfies the smoothness conditions of Section 2 (see A.III). Note that, for each fixed value of the first argument, $\left\{W_{n l}(\cdot, v) / W_{n}^{*}(\cdot)\right\}$ is assumed to be a "Heaviside" sequence.

In order to establish the main results (Theorems 3.1 and 3.2) of this section, we need the following expansion of $v_{n x}(y)$ which is valid in view of the smoothness assumptions A.III(i), (ii) above: For each $x \in \Lambda(F)$ and $y \in \mathscr{R}$, we have

$$
\begin{aligned}
v_{n x}(y)= & t_{n}(x)\left[G_{n x}(y)-G_{x}(y)\right] \\
= & a_{n}^{-1} \iint_{A_{n}}\left[W_{n}\left((F(x)-F(u)) / a_{n}, y-v\right)\right. \\
& \left.\quad-W_{n}^{*}\left((F(x)-F(u)) / a_{n}\right) G_{x}(y)\right] d H_{n}(u, v) \\
+ & n^{-\frac{1}{2}} a_{n}^{-2} \iint_{A_{n}}\left[U_{n}(x)-U_{n}(u)\right]\left[W_{n}^{(1.0)}\left((F(x)-F(u)) / a_{n, y} y\right)\right. \\
& \left.\quad-W_{n}^{*(1)}\left((F(x)-F(u)) / a_{n}\right) G_{x}(y)\right] d H_{n}(u, v) \\
+ & 2^{-1} n^{-1} a_{n}^{-3} \iint_{A_{n}}\left[U_{n}(x)-U_{n}(u)\right]^{2}\left[W_{n}^{(2.0)}\left(\Delta_{n}, \mathrm{y}-v\right)\right. \\
& \left.\quad-W_{n}^{*(2)}\left(\Delta_{n}\right) G_{x}(y)\right] d H_{n}(u, v) \\
= & J_{n 1}(v)+J_{n 2}(y)+J_{n 3}(y) \quad(\text { say }),
\end{aligned}
$$

where $A_{n}=\left\{u:\left|F_{n}(x)-F_{n}(u)\right| \leq a_{n}\right\}, W_{n}^{\left(j, j^{\prime}\right)}(t, s)=\left(j, j^{\prime}\right)$ th partial derivative of $W_{n}(t, s)$, and $a_{n} \Delta_{n}$ lies between $[F(x)-F(u)]$ and $\left[F_{n}(x)-F_{n}(u)\right]$.

We first prove two results in Lemma 3.1 below concerning the asymptotic behavior of $J_{n j}(y), j=2,3$.

Lemma 3.1. Let $x \in \Lambda(F)$ be fixed. Suppose the assumptions A.I, A.II and $A . I I I(i)$, (ii) and (v) and $A . I V(i)$ and (ii) hold. Then as $n \rightarrow x$, uniformly in $y \in \mathscr{R}$ (a)

$$
J_{n j}(y) \underset{\text { a.s. }}{=} o\left(\tau_{n}\right), j=2,3,
$$

where $\tau_{n}=\left(n a_{n}\right)^{-1 / 2}\left(\log a_{n}^{-1}\right)^{1 / 2}$ and

$$
J_{n j}(y)=o_{p}\left(n^{-\frac{1}{2}} a_{n^{-\frac{1}{2}}}\right), j=2,3 .
$$

Proof. We first deal with $J_{n 3}(y)$. Since $W_{n}$ vanishes for values of the first argument outside $[-1,1]$, the expansion (3.2) holds with integration restricted to the set $A_{n}$ $=\left\{u:\left|F_{n}(x)-F_{n}(u)\right| \leq a_{n}\right\}$, and further by Proposition 2.1(i), we have almost surely on this set

$$
\begin{aligned}
|F(x)-F(u)| & \leq\left|F_{n}(x)-F_{n}(u)\right|+n^{-\frac{1}{2}}\left|U_{n}(x)-U_{n}(u)\right| \\
& \leq a_{n}+C_{1}\left(a_{n} \log a_{n}^{-1} / n\right)^{\frac{1}{2}} \leq C a_{n}
\end{aligned}
$$

for some constants $C_{1}$ and $C$ and sufficiently large $n$, the last inequality following in view of A.IV(ii). Now writting $p_{n}(y, u, v)=\left[W_{n}^{(2.0)}\left(\Delta_{n}, y-v\right)-W_{n}^{*(2)}\left(\Delta_{n}\right) G_{x}(y)\right]$, we have 


$$
\begin{aligned}
J_{n .3}(y)= & 2^{-1} n^{-1} a_{n}^{-3} \iint_{A_{n}}\left[U_{n}(x)-U_{n}(u)\right]^{2} p_{n}(y, u, v) d\left[H_{n}(u, v)-H(u, v)\right] \\
& +2^{-1} n^{-1} a_{n}^{-3} \iint_{A_{n}}\left[U_{n}(x)-U_{n}(u)\right]^{2} p_{n}(y, u, v) d H(u, v) \\
= & J_{n, 31}(y)+J_{n, 32}(y) \text { (say). }
\end{aligned}
$$

From Proposition 2.1, the boundedness of $p_{n}(y, u, v)$ and (3.3) we have, as $n \rightarrow x$

$$
\begin{aligned}
\left|J_{n .31}(y)\right| & \leq C_{1} n^{-1} a_{n}^{-3} \iint_{\mathcal{A}_{n}}\left[U_{n}(x)-U_{n}(u)\right]^{2} d\left|H_{n}(u, v)-H(u, v)\right| \\
& \leq C_{1} n^{-1} a_{n}^{-3} \sup _{|F(x)-F(u)| \leq C a_{n}}\left|U_{n}(x)-U_{n}(u)\right|^{2} \sup _{u . v}\left|H_{n}(u, v)-H(u, v)\right| \\
& \leq C_{1} n^{-1} a_{n}^{-3} \cdot a_{n} \log a_{n}^{-1} n^{-\frac{1}{2}}(\log \log n)^{\frac{1}{2}} \\
& =O\left(\tau_{n}\left(n a_{n}^{3}\right)^{-1} a_{n}^{\frac{3}{2}}\left(\log a_{n}^{-1}\right)^{\frac{1}{2}}(\log \log n)^{\frac{1}{2}},\right.
\end{aligned}
$$

where $A_{n}^{*}=A_{n} \cap\left\{|F(x)-F(u)| \leq C a_{n}\right\}$. As for $J_{n 32}$, we note that in view of the boundedness of $p_{n}(y, u, v),(3.3)$ and Proposition 2.1, as $n \rightarrow \infty$,

$$
\begin{aligned}
\left|J_{n 32}(y)\right| & \leq n^{-1} a_{n}^{-3} C_{1} \sup _{|F(n)-F(u)| \leq C a_{n}}\left|U_{n}(x)-U_{n}(u)\right|^{2} \int_{\mid F(x)-F(u) \leq C\left(a_{n}\right.} d F(u) \\
& \leq C_{1} n^{-1} a_{n}^{-3}\left(a_{n} \log a_{n}^{-1}\right) \cdot C a_{n} \\
& \leq C_{2} \tau_{n}^{2},
\end{aligned}
$$

the last but one inequality following from the fact that $P\left[|F(X)-F(u)| \leq C a_{n}\right]=$ $2 C a_{n}$. From (3.4), (3.5) and (3.6), in view of assumptions A.IV (i) and (ii), we thus obtain

$$
\left|J_{n 3}(y)\right| \underset{\text { a.s. }}{=} o\left(\tau_{n}\right)
$$

Now for $J_{n 2}(y)$, note that it can be expressed as

$$
\begin{aligned}
J_{n 2}(y)= & n^{-\frac{1}{2}} a_{n}^{-2} \iint_{A_{n}}\left[U_{n}(x)-U_{n}(u)\right]\left[W_{n}^{(1.0)}\left((F(x)-F(u)) / a_{n}, y-v\right)\right. \\
& \left.-W_{n}^{*(1)}\left((F(x)-F(u)) / a_{n}\right) G_{x}(y)\right] d H(u, v) \\
+ & n^{-\frac{1}{2}} a_{n}^{-2} \iint_{A_{n}^{*}}\left[U_{n}(x)-U_{n}(u)\right]\left[W_{n}^{(1.0)}\left((F(x)-F(u)) / a_{n}, y-v\right)\right. \\
& \left.-W_{n}^{*(1)}\left((F(x)-F(u)) / a_{n l}\right) G_{x}(y)\right] d\left[H_{n}(u, v)-H(u, v)\right] \\
= & J_{n 21}(y)+J_{n 22}(y) \quad \text { (say), }
\end{aligned}
$$

where, using the boundedness of functions $W_{n}$ and $W_{n}^{*}$, and Proposition 2.1, it can be shown as for $J_{n 31}(y)$ that

$$
\begin{aligned}
\left|J_{n 22}(y)\right| & \leq C_{1} n^{-1} a_{n}^{-2} \sup _{u \in A_{n}}\left|U_{n}(x)-U_{n}(u)\right| \sup _{(u, v)} n^{\frac{1}{2}}\left|H_{n}(u, v)-H(u, v)\right| \\
& \leq C_{1} n^{-1} a_{n}^{-\frac{3}{2}}\left(\log a_{n}^{-1}\right)^{\frac{1}{2}}(\log \log n)^{\frac{1}{2}}
\end{aligned}
$$




$$
=o\left(\tau_{n}\right) \text {, }
$$

as $n \rightarrow \infty$, in view of the assumptions A.IV (i) and (ii). Further, making the transformation $F(x)-F(u)=a_{n} t$ and setting $x_{n}(t)=F^{-1}\left(F(x)-t a_{n}\right)$, we obtain for $J_{n 21}(y)$

$$
\begin{aligned}
\left|J_{n 21}(y)\right|= & n^{-\frac{1}{2}} a_{n}^{-1} \mid \iint_{A_{n}^{*}}\left[U_{n}(x)-U_{n}\left(x_{n}(t)\right)\right]\left[W_{n}^{(1,0)}(t, y-v)\right. \\
& \left.-W_{n}^{*(1)}(t) G_{.}(y)\right] d G_{x_{n}(t)}(v) d t \mid \\
\leq & C \tau_{n}\left[\int_{A_{i n}^{*}}\left|\int\left[W_{n}^{(1,0)}(t, y-v)-W_{n}^{*(1)}(t) G_{x}(y)\right] d G_{x}(v)\right| d t\right. \\
& \left.+\int_{\mathcal{A}_{n}^{*}}\left|\int\left[W_{n}^{(1,0)}(t, y-v)-W_{n}^{*(1)}(t) G_{x}(y)\right] d\left[G_{x_{n}(t)}(v)-G_{x}(v)\right]\right| d t\right] \\
= & o\left(\tau_{n}\right), \text { as } n \rightarrow \infty,
\end{aligned}
$$

the last inequality following in view of Proposition 2.1 and the last equality since the preceding integrals are $o(1)$ and $O\left(a_{n}\right)$, respectively, as $n \rightarrow \infty$, in view of (3.3) and the assumption A.III(v). From (3.8), (3.9) and (3.10), it follows that, as $n \rightarrow \infty$, with probability one

$$
J_{n 2}(y)=o\left(\tau_{n}\right)
$$

The proof of part (a) is complete in view of (3.7) and (3.11)

To prove part (b), we use the facts that $n^{\frac{1}{2}}\left[H_{n}(u, v)-H(u, v)\right]=O_{p}(1)$ and $\sup \left\{\left|U_{n}(x)-U_{n}(u)\right|:|F(x)-F(u)| \leq C a_{n}\right\}=O_{p}\left(a_{n}^{\frac{1}{2}}\right)$ (see Proposition 2.1(ii)). Using these probability bounds, we have from the second inequality in (3.5)

$$
\begin{aligned}
\left|J_{n 31}(y)\right| & \leq{ }_{p} C_{1} n^{-1} a_{n}^{-3}\left(a_{n}^{\frac{1}{2}}\right)^{2} \cdot n^{-\frac{1}{2}} \\
& =C_{1} n^{-\frac{1}{2}} a_{n}^{-\frac{1}{2}}\left(n^{-1} a_{n}^{-\frac{3}{2}}\right) \\
& =o_{p}\left(n^{-\frac{1}{2}} a_{n}^{-\frac{1}{2}}\right)
\end{aligned}
$$

this together with a similar analogue of (3.6) implies $J_{n 32}(y)=O_{p}\left(n^{-\frac{1}{2}} a_{n}^{-\frac{1}{2}}\right)$. Thus

$$
J_{n 3}(y)=o_{p}\left(n^{-\frac{1}{2}} a_{n}^{-\frac{1}{2}}\right) .
$$

By using probability bounds instead of a.s. bounds as for (3.11), it can be similarly seen that

$$
J_{n 2}(y)=o_{p}\left(n^{-\frac{1}{2}} a_{n}^{-\frac{1}{2}}\right)
$$

and this completes the proof of part (b).

We now state the main theorem concerning a.s. convergence rates and the asymptotic normality of the estimators $\widehat{G}_{n x}$ and $\widehat{G}_{n x}^{*}$ :

THEOREM 3.1. Let $x \in \Lambda(F)$ be fixed and suppose that the assumptions of Lemma 3.1 hold.

(a) If in addition to the assumptions of Lemma 3.1, A.III(iii) and (iv) also hold for some $m \geq 1$, then for each $y \in \mathscr{R}$ 


$$
\left|\widehat{G}_{n x}(y)-G_{x}(y)\right|=O\left(\tau_{n}\right), \text { a.s., as } n \rightarrow x,
$$

where $\tau_{n}$ is as defined in Lemma 3.1; and further

(b) if in addition to the assumptions of Lemma 3.1, A.IV (iii) also holds, then for each $y \in \mathscr{R}$,

$$
n^{\frac{1}{2}} a_{\bar{n}}^{\frac{1}{2}}\left(\widehat{G}_{m x}(y)-G_{x}(y)\right) \underset{\mathcal{L}}{\longrightarrow} N\left(0, \sigma_{x}^{2}(y)\right) \text {, as } n \rightarrow x
$$

where $\sigma_{x}^{2}(y)=G_{x}(y)\left(1-G_{x}(y)\right)\left(\int k_{1}^{2}(t) d t\right)$ and $k_{1}$ is defined by $A . I I I(v)$.

Proof. We shall first establish part (a). In view of Lemma 3.1(a), it suffices to prove for $J_{n 1}$ and $t_{n}(x)$ in (3.2) that, as $n \rightarrow \infty$, for each given $x \in \Lambda(F), t_{n}(x) \rightarrow 1$ a.s. and for each $y \in \mathscr{R}$,

$$
\left|J_{n 1}(y)\right| \underset{\text { i... }}{=} O\left(\tau_{n}\right)
$$

To see that (3.12) holds, we first note that, since for each $y \in \Re$,

$$
\left|J_{n 1}(y)-\bar{J}_{n 1}(y)\right|=O\left(\tau_{n}\right) \text { a.s. }
$$

as $n \rightarrow \infty$ (to be established; see (3.23) below) where $\bar{J}_{n 1}(y)$ is just $J_{n 1}(y)$ with integration over the whole space instead of the set $A_{n}$, it suffices to establish (3.12) with $J_{n 1}(y)$ replaced by $\bar{J}_{n 1}(y)$. To achieve this, we shall write $\bar{J}_{n 1}(y)=n^{-1} \sum_{i=1}^{n} Z_{n i}$ with

$$
Z_{n i}=a_{n}^{-1}\left[W_{n}\left(\left(F(x)-F\left(X_{i}\right)\right) / a_{n}, y-Y_{i}\right)-W_{\mathrm{n}}^{*}\left(\left(F(x)-F\left(X_{i}\right) / a_{n}\right) G_{x}(y)\right],\right.
$$

$1 \leq i \leq n$, so that by using the transformation $F(x)-F(u)=a_{n} t$ and setting $x_{n}(t)=$ $F^{-1}\left(F(x)-t a_{n}\right)$ in below, we obtain, in view of assumption A.I.

$$
\begin{aligned}
E\left(Z_{n i}\right)= & \iint\left[W_{n}(t, y-v)-W_{n}^{*}(t) G_{x}(y)\right] d G_{x_{n}(t)}(v) d t \\
= & \iint\left[W_{n}(t, y-v)-W_{n}^{*}(t) G_{x}(y)\right] d G_{x}(v) d t \\
& +\iint\left[W_{n}(t, y-v)-W_{\mathrm{n}}^{*}(t) G_{x}(y)\right] d\left[G_{x_{n}(t)}(v)-G_{x}(v)\right] d t \\
= & \int\left[\int W_{n}(t, y-v) d t-G_{x}(y)\right] d G_{x}(v)+O\left(a_{n}^{m+1}\right)
\end{aligned}
$$

that the second term in the last expression in (3.13) is $O\left(a_{n}^{m+1}\right)$ uniformly in $y$ follows by using Taylor's expansion of $G_{x_{n}(t)}(v)$ around $G_{x}(v)$ in conjunction with assumptions A.I, A.II and A.III(i), (iii). Now the integral term in this last expression equals, using A.I, A.III(i), (iii) and integration by parts,

$$
\begin{aligned}
& \left|\int m_{n}^{*}(y-v) d G_{x}(v)-G_{x}(y)\right| \\
& \quad=\left|\int\left[G_{x}(y-v)-G_{x}(y)\right] d m_{n}^{*}(v)\right|
\end{aligned}
$$




$$
\begin{aligned}
& =\mid \int\left[v^{m+1} /(m+1) !\right] \frac{\partial^{m+1}}{\partial v^{m+1}}\left(G_{x}\left(y-v^{*}\right) d m_{n}^{*}(v) \mid\right. \\
& \leq C_{1} \int|v|^{m-1} d m_{n}^{*}(v),
\end{aligned}
$$

where $v^{*}$ lies between 0 and $v, m_{n}^{*}(v)=\int W_{n}(t, v) d t$ and $C_{1}$ is a constant not depending on $n$. Further in view of the assumptions A.III(ii) and (iv) we have

$$
\begin{aligned}
\int|v|^{m+1} d m_{n}^{*}(v) & =\left.\left|\int_{|v| \leq b_{n}}\right| v\right|^{m+1} d m_{n}^{*}(v)+\int_{|v| \geq h_{n}}|v|^{m+1} d m_{n}^{*}(v) \\
& =O\left(b_{n}^{m+1}\right)+O\left(b_{n}^{m+1}\right) \\
& =O\left(\tau_{n}\right)
\end{aligned}
$$

as $n \rightarrow x$. From (3.13) to (3.15) and the assumption A.III (iv), we thus have

$$
E\left(Z_{n i}\right)=O\left(\tau_{n}\right) .
$$

Further, again by assumptions A.I, A.II and A.III(i), (ii) and (v), and the transformation used for (3.13) we obtain

$$
\begin{aligned}
a_{n} E\left(Z_{n i}^{2}\right)= & \iint\left[W_{n}(t, y-v)-W_{n}^{*}(t) G_{x}(y)\right]^{2} d G_{x_{n}(t)}(v) d t \\
= & \iint\left[W_{n}^{2}(t, y-v)+W_{n}^{* 2}(t) G_{x}^{2}(y)\right. \\
& \left.-2 W_{n}(t, y-v) W_{n}^{*}(t) G_{x}(y)\right] d G_{x}(v) d t+O\left(a_{n}\right) \\
\rightarrow & \iint\left[k_{1}^{2}(t) I_{[y-v \geq 0]}+k_{1}^{2}(t) G_{x}^{2}(y)-2 k_{1}^{2}(t) I_{[y-v \geq 0]} G_{x}(y)\right] d G_{x}(v) d t \\
= & G_{x}(y)\left[1-G_{x}(y)\right]\left(\int k_{1}^{2}(t) d t\right) .
\end{aligned}
$$

Now noting that $\left|Z_{n i}\right| \leq a_{n}^{-1}$ for all $1 \leq i \leq n$ and using (3.17) and Bernstein inequality (see Serfling [12] pp. 98-99), we obtain, for any $\varepsilon>0$ and some constant $C_{1}>0$,

$$
\begin{aligned}
P\left[\left|\sum_{i=1}^{n}\left[Z_{n i}-E\left(Z_{n i}\right)\right]\right|\right. & \geq n \varepsilon] \leq 2 \exp \left\{-\frac{n \varepsilon^{2}}{\left[\frac{1}{n} \sum_{i=1}^{n} \operatorname{var}\left(Z_{n i}\right)+(2 / 3) a_{n}^{-1} \varepsilon\right]}\right\} \\
& \leq 2 \exp \left\{\frac{-n a_{n} \varepsilon^{2}}{C_{1}+(2 / 3) \varepsilon}\right\}
\end{aligned}
$$

which inequality, with $\varepsilon=C_{2} \tau_{n}$, yields

$$
\begin{aligned}
P\left[\left|n^{-1} \sum_{i=1}^{n}\left[Z_{n i}-E\left(Z_{n i}\right)\right]\right|\right. & \left.\geq C_{2} \tau_{n}\right] \\
& \leq 2 \exp \left\{\frac{-C_{2}^{2}\left(\log a_{n}^{-1}\right)}{C_{1}+(2 / 3) \tau_{n}}\right\} .
\end{aligned}
$$


Now using the assumption A.IV(ii) (which implies for sufficiently large $n$ the existence of a constant $C_{3}$ such that $a_{n}^{-1}>C_{3} n^{\frac{1}{*}}$ and $\tau_{n} \rightarrow 0$ as $\left.n \rightarrow \infty\right)$ and choosing $C_{1}$, $C_{2}, C_{3}$ appropriately so that R.H.S. of (3.18) is dominated by a constant multiple of $n^{-2}$ for sufficiently large $n$, it follows in view of Borel-Cantelli lemma that

$$
\left|\bar{J}_{n 1}(y)-E\left(\bar{J}_{n 1}(y)\right)\right|=\frac{1}{n} \sum_{i=1}^{n}\left[Z_{n i}-E\left(Z_{n i}\right)\right] \underset{\text { a.s. }}{=} O\left(\tau_{n}\right) .
$$

From (3.16) and (3.19) then, we obtain

$$
\left|\bar{J}_{n 1}(y)\right| \underset{\substack{\text { a.s. }}}{=} O\left(\tau_{n l}\right)
$$

as $n \rightarrow x$. To conclude (3.20) for $J_{n 1}(y)$, we now establish the asymptotic a.s. equivalence (3.12a): First note that on the set $A_{n}^{c} \cap A_{n}^{* *}$, where $A_{n}^{* * *}=\left\{u:|F(x)-F(u)| \leq a_{n}\right\}$, for sufficiently large $n$

$$
\begin{aligned}
a_{n} \geq|F(x)-F(u)| & =\left|F_{n}(x)-F_{n}(u)-n^{-\frac{1}{2}}\left\{U_{n}(x)-U_{n}(u)\right\}\right| \\
& \geq a_{\text {a.s. }}\left(1-\varepsilon_{n}\right),
\end{aligned}
$$

the last inequality following since by Proposition 2.1(i) on $A_{n}^{* *}$, uniformly in $u, \mid U_{n}(x)$ $-U_{n}(u) \mid \leq c_{2} a_{n}{ }^{1 / 2}\left(\log a_{n}^{-1}\right)^{1 / 2}=a_{n} \varepsilon_{n}$ a.s. with $\varepsilon_{n}=c_{2} \tau_{n}$ for some constant $c_{2}>0$, as $n \rightarrow \infty$. From (3.21), we obtain for each $y \in \mathscr{R}$ on using transformation $F(x)-F(u)=$ $a_{n l} t,-1 \leq t \leq 1$, and on setting $u=x_{n}(t)=F^{-1}\left(F(x)-a_{n} t\right)$

$$
\begin{aligned}
\left|J_{n 1}(y)-\bar{J}_{n 1}(y)\right| \leq & \int\left|\int_{\left[1-\varepsilon_{n} \leq|r| \leq 1 \mid\right.}\left[W_{n}(t, y-v)-W_{n}^{*}(t) G_{x}(y)\right] d G_{x_{n}(t)}(v)\right| d t \\
+ & a_{n}^{-1} \int_{\left|1-\varepsilon_{n} \leq\right| t|\leq 1|}\left[W_{n}(t, y-v)-W_{n}^{*}(t) G_{x}(y) \mid\right. \\
& d\left|H_{n}\left(x_{n}(t), v\right)-H\left(x_{n}(t), v\right)-H_{n}\left(x_{n}(1), v\right)+H\left(x_{n}(1), v\right)\right| \\
\leq & 2 \varepsilon_{n}\left|\int\left[W_{n}\left(t_{n}^{*}, y-v\right)-W_{n}^{*}\left(t_{n}^{*}\right) G_{x}(y)\right] d G_{x_{n}\left(t_{n}\right)}(v)\right| \\
+ & c_{3} n^{-\frac{1}{2}} a_{n}^{-1}\left[\left|U_{n}\left(x_{n}\left(1-\varepsilon_{n}\right)\right)-U_{n}\left(x_{n}(1)\right)\right|\right. \\
& \left.+\left|U_{n}\left(x_{n}(-1)\right)-U_{n}\left(x_{n}\left(\varepsilon_{\mathbf{n}}-1\right)\right)\right|\right] \\
= & O\left(\tau_{n}\right), \\
\text { a. } .5 &
\end{aligned}
$$

as $n \rightarrow \infty$, where $1-\varepsilon_{n} \leq\left|t_{n}^{*}\right| \leq 1$, the last inequality following since $\varepsilon_{n}=c_{2} \tau_{n}$ the integral in the first term of the preceding expression is $O(1)$ (cf. (3.13)) as $n \rightarrow \infty$, and the second term, by Proposition 2.1(i), is $O\left(n^{-\frac{1}{2}} a_{n}^{-1} a_{n}^{\frac{1}{2}} \frac{1}{2}\left(\log \left(a_{n} \varepsilon_{\mathrm{n}}\right)^{-1}\right)^{\frac{1}{2}}\right)=o\left(\tau_{n}\right)$ a.s., as $n \rightarrow \infty$, under conditions A.IV (i) and (ii) of the Theorem. The assertion (3.12) now follows from (3.20) to (3.22). Accordingly, from Lemma 3.1(a), (3.2) and (3.12), the proof of part (a) of the theorem would be complete if we show that

$$
\left|t_{n}(x)-1\right|=O\left(\tau_{n}\right)
$$

with probability one, as $n \rightarrow \infty$. The last assertion, however, follows (evidently) by the 
same reasoning as for Lemma 3.1(a) and (3.20). This completes the proof of part (a).

To prove that (b) concerning the asymptotic normality of $n^{\frac{1}{2}} a_{n}^{\frac{1}{2}}\left[G_{n x}(y)-G_{x}(y)\right]$, as $n \rightarrow \infty$, it suffices to show that $n^{\frac{1}{2}} a_{h l}^{\frac{1}{2}} J_{n 1}(y) \longrightarrow N\left(0, \sigma_{x}^{2}(y)\right)$; the proof would then be complete in view of Lemma 3.1(b). Now by following arguments similar to those for (3.21) to (3.22), we can show that $\left|J_{n 1}(y)-\bar{J}_{n 1}(y)\right|=O_{p}\left(n^{-\frac{i}{2}} a^{-\frac{1}{2}}\right)$ as $n \rightarrow \infty$. Also from (3.13) to (3.17), we have $\bar{J}_{n 1}(y)=n^{-1} \sum_{i=1}^{n} Z_{n i}, Z_{n i}$ being independent and identically distributed with $E\left(Z_{n i}\right)=O\left(b_{n}^{m+1}\right)$ and $a_{n} E\left(Z_{n i}^{2}\right) \rightarrow \sigma_{x}^{2}(y)=G_{x}(y)\left(1-G_{x}(y)\right)$ $\left(\int k_{1}^{2}(t) d t\right)$. Since $n^{\frac{1}{2}} a_{n}^{\frac{1}{2}} b_{n}^{m+1} \rightarrow 0$, as $n \rightarrow \infty$, (A.IV (iii)), it follows by the standard Central Limit Theorem that $n^{\frac{1}{2}} a_{\bar{n}}^{\frac{1}{2}} J_{n 1}(y) \longrightarrow N\left(0, \sigma_{x}^{2}(y)\right)$, as $n \rightarrow x$. This completes the proof of part (b)

REMARK 3.1. If $m_{n}^{*}(\cdot)=\int W_{n}(t, \cdot) d t$ is smooth and possesses a bounded derivative, then in Theorem 3.1 (and also in Theorem 3.2 below) the condition A.III(iv) above can be replaced by the weaker condition (say) A.III(vi): $\int_{|v| \geq b_{n}}|v|^{m} d m_{n}^{*}(v)=O\left(b_{n}^{m+1}\right)$ (with $b_{n}$ as given in A.III(iv)) on the tails of $m_{n}^{*}$. However, this would not cover the case of unsmoothed c.e.d.f.'s. It should be noted, nevertheless, that unsmoothed c.e.d.f.'s and the smoothed ones with bivariate kernels (product or not) of the type $W_{n}(t, s)=W\left(t, \frac{s}{a_{n}}\right)$ (see Section 4) do satisfy the stronger assumption A.III(iv). Consequently, our special comparisons of smoothed and unsmoothed c.e.d.f.'s in Section 4 remain valid.

Now we will show that even if $a_{n}$ (or $b_{n}$ ) $\rightarrow 0$ at the so called "optimal" rate (see Remark 4.1) i.e., for $m=1$ even if $n a_{n}^{5}$ or more generally, $n a_{n}^{2 m+3} \rightarrow \theta$ a positive constant and not zero, the asymptotic normality of Theorem 3.1(b) still holds with a non-zero centering constant. However, we need some additional conditions on the kernel function $W$ :

Corollary 3.1. Suppose in addition to $A . I V(i)$ and (ii), we have $n a_{n}^{2 m+3} \rightarrow \theta \geq 0$, as $n \rightarrow \infty$, and that $m_{n}^{*}$ (as defined in A.III(iv)) satisfies for some $\theta^{*},-\infty<\theta<x$,

$$
\int t^{m+1} d m_{n}^{*}\left(a_{n} t\right) \rightarrow \theta^{*} \text {, as } n \rightarrow \infty .
$$

(which impies the condition $\int s^{m+1} d m_{n}^{*}(s)=O\left(a_{n}^{m+1}\right)$ resulting from $\left.A . I I I(i v)\right)$. Then under the assumptions A.I, A.II and A.III,

$$
\left(n a_{n}\right)^{\frac{1}{2}}\left(\widehat{G}_{n . x}(y)-G_{x}(y)\right) \underset{\mathcal{L}}{\longrightarrow} N\left(b_{x}(y), \sigma_{x}^{2}(y)\right),
$$

as $n \rightarrow \infty$, where $\sigma_{x}^{2}(y)$ is as given in Theorem $3.1(b)$ and $b_{x}(y)$ by

$$
\begin{aligned}
b_{x}(y)= & (\sqrt{\theta} /(m+1) !)\left\{\theta^{*} \frac{\partial^{m+1}}{\partial y^{m+1}}\left[G_{x}(y)\right]\right. \\
& \left.+\left(\int t^{m+1} k_{1}(t) d t\right) \frac{\partial^{m+1}}{\partial \eta^{m+1}}\left[G_{F^{-1}(\eta)}(y)\right]_{\eta=F(x)}\right\},
\end{aligned}
$$

where $k_{1}$ is as defined in $A I I I(v)$. 
Proof. Since Lemma 3.1(b) still holds, the proof of Theorem 3.1(b) shows that we need only to demonstrate that $\left(n a_{n}\right)^{1 / 2} E J_{n 1}(y)=\left(n a_{n}\right)^{1 / 2} E\left(Z_{n 1}\right) \rightarrow b_{x}(y)$ under the stated conditions of the corollary, where $Z_{n 1}$ is defined by $(3.12 \mathrm{~b})$ : We have from (3.13) by setting $G_{F^{-1}(\lambda)}(v)=G_{\lambda}^{*}(v)$ and using A.III(iii)

$$
\begin{aligned}
E Z_{n 1}= & \iint\left[W_{n}(t, y-v)-W_{n}^{*}(t) G_{x}(y)\right] d G_{F(x)-a_{n}}^{*}(v) d t \\
= & \iint\left[W_{n}(t, y-v)-W_{n}^{*}(t) G_{x}(y)\right] d G_{F(x)}^{*}(v) d t \\
& +\left(a_{n}^{m+1} /(m+1) !\right) \iint\left[W_{n}(t, y-v)-W_{n}^{*}(t) G_{x}(y)\right] t^{(m+1)} d G_{x_{t, n}^{*}}^{(m+1)}(v) \mathrm{dt} \\
= & I+I I \quad \text { (say), }
\end{aligned}
$$

where $G_{x_{m}}^{*(m+1)}(v)=\left.\frac{3^{m+1}}{3 \eta^{\prime \prime+1}} G_{\eta}^{*}(v)\right|_{\eta=x_{i n}}$ etc., $x_{o n}$ lies between $F(x)$ and $F(x)-a_{n} t$, and the second equality is obtained by Taylor's expansion of $G_{F(x)-a_{l} t}^{* *}(v)$ around $G_{F(x)}^{*}(v)$. Now following steps similar to those for (3.14), it is easily seen that as $n \rightarrow \infty$

$$
\begin{aligned}
a_{n}^{-(m+1)} I & =(1 /(m+1) !) \frac{\partial^{m+1}}{\partial y^{m+1}}\left[G_{x}(y)\right] \int s^{m+1} d m_{n}^{*}\left(a_{n} s\right) \\
& \rightarrow(1 /(m+1) !) \frac{\partial^{m+1}}{\partial y^{m+1}}\left[G_{x}(y)\right] \theta^{*}
\end{aligned}
$$

Further, using the limits $W_{n}^{*}(t) \rightarrow k_{1}(t)$ and $\left[W_{n}(t, y-v) / W_{n}^{*}(t)\right] \rightarrow I_{[y-1 \geq 0]}$ as $n \rightarrow x$, we obtain that, as $n \rightarrow x$,

$$
\begin{aligned}
& \iint t^{m+1} {\left[W_{n}(t, y-v)-W_{n}^{*}(t) G_{x}(y)\right] d G_{x_{m}}^{*(m+1)}(v) d t } \\
& \rightarrow\left(\int t^{m+1} k_{1}(t) d t\right)\left\{\frac{\partial^{m+1}}{\partial \eta^{m+1}}\left[G_{F^{-1}(\eta)}(y)\right]_{\eta=F(x)}\right\} .
\end{aligned}
$$

From (3.26) to (3.28) we thus have under the assumptions of the corollary that $\left(n a_{n}\right)^{1 / 2}$ $E Z_{n 1} \rightarrow b_{x}(y), n \rightarrow \infty$, as asserted.

REMARK 3.2. The bias term $b_{x}(y)$ above involves the $(m+1)^{t h}$ order partial derivatives of $G_{x}(y)$ both w.r. to $y$ and w.r. to $x$. It should be noted that the $(m+1)^{t h}$ order partial derivative in the second term of the bias for $m=1$ equals

$$
\left.\frac{\partial^{2}}{\partial \eta^{2}} G_{F^{-1}(\eta)}(y)\right|_{\eta=F(x)}=\left\{f(x) \frac{\partial^{2}}{\partial x^{2}}\left[G_{x}(y)\right]-f^{\prime}(x) \frac{\partial}{\partial x}\left[G_{x}(y)\right]\right\} / f^{3}(x) .
$$

REmark 3.3. In Theorem 3.1 and Corollary 3.1, we have assumed the same order $m$ for the kernel $W_{n}(\cdot, \cdot)$ in either argument (cf. A.III(iii)). If we choose different orders, say, $m_{1}$ and $m_{2}$ for the first and second arguments, respectively, then on evaluating $E\left[Z_{n 1}\right]$ in (3.13) the order of $E\left(Z_{n 1}\right)$ in (3.16) would be $O\left(\tau_{n n^{2}}^{\frac{m+1}{2}}\right)$ with $m=$ $m_{1} \wedge m_{2}$. Further in Corollary 3.1, the asymptotic bias $b_{x}(y)$ would be $b_{x 1}=\left[\sqrt{\theta} /\left(m_{2}+\right.\right.$ 


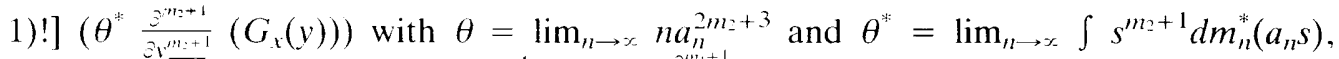
$b_{x 2}=\left.\left(\sqrt[3]{\theta^{\prime}} /\left(m_{1}+1\right) !\right)\left(\int t^{m_{1}+1} k_{1}(t) d t\right) \frac{3^{m_{1}+1}}{3 y^{\prime \prime+1}}\left[G_{F^{-1}(\eta)}(y)\right]\right|_{\eta=F(x)}$ with $\theta^{\prime}=\lim _{n \rightarrow \infty}$ $n a_{n}^{2 m_{1}+3}$ or $\left[b_{x 1}+b_{x 2}\right]$ according as $m_{2}<m_{1}, m_{2}>m_{1}$ or $m_{1}=m_{2}$. The remarks above also apply to the results of Section 4 dealing with deficiency calculations.

REMARK 3.4. If $m=1$, and smoothing is not employed (as in Stute [16]), we then have $m_{n}^{*}(s)=I_{[s \geq 0]}$ so that $\int s^{2} d m_{n}^{*}\left(a_{n n} s\right)=0$; and the bias in this case reduces to

$$
\frac{\sqrt{\theta}}{2}\left(\int t^{2} k_{1}(t)\right) \frac{\partial^{2}}{\partial \eta^{2}}\left[G_{F^{-1}(\eta)}(y)\right]_{\eta=F(x)},
$$

the same as given in Stute (1986a, p. 641).

Furthermore, if $W_{n}(t, s)=W\left(t, s / a_{n}\right)$ for some bivariate kernel function as defined in $(1.1)$, then $\int s^{m+1} d m_{n}^{*}\left(a_{n} s\right)=\int s^{m+1} d K_{2}(s)$, with $K_{2}(s)=\int W(t, s) d t$. Accordingly the case of product kernels considered in literature (see Section 4 ) is also covered by our results.

REMARK 3.5. In defining $\widehat{G}_{n x}$, if we take the kernels $W_{n}$ to be non-negative and non-decreasing in the second argument, then $\widehat{G}_{n x x}$ satisfies all properties required of a probability d.f., namely, (i) $0 \leq \widehat{G}_{n x}(y) \leq 1 \forall y$, (ii) non-decreasing in $y$, and (iii) $\lim _{y \rightarrow-\infty} \widehat{G}_{n x}(y)=0$ and $\lim _{y \rightarrow \infty} \widehat{G}_{n x}(y)=1$. For $\widehat{G}_{n x}$ based on higher order kernels, the first two properties may not hold for a given set of observations. However, in view of the result (Theorem 3.1) that $\widehat{G}_{n x}(y) \underset{\text { i.s. }}{\longrightarrow} G_{x}(y)$, with $0 \leq G_{x}(y) \leq 1$, as $n \rightarrow x$, at a rate no slower than $\tau_{n}=n^{-\frac{1}{2}} a_{n^{-\frac{1}{2}}}\left(\log a_{n}^{-1}\right)^{1 / 2} \rightarrow 0$, it follows that, on a set $S$ of arbitrarily high probability, $\widehat{G}_{n \cdot x}$ will be close to satisfying these properties for sufficiently large $n$ say, for $n \geq n_{0}$, with $n_{0}$ not depending on $w \in S$.

Now we state a result analogous to Theorem 3.1 for the NW type estimate $\widehat{G}_{\mathrm{nx}}^{*}$ of the conditional d.f. $G_{x}$. The proof is, in fact, contained in the proof of Theorem 3.1 and is hence omitted.

Theorem 3.2. Suppose $x \in \Lambda(F)$ is fixed and the assumptions A.I, A.II, A.III $(i)-(v)$ and $A . I V(i)$, (ii) and (iii) hold. Then as $n \rightarrow \infty$, with $\tau_{n}$ as defined in Lemma 3.1,

(a) $\left|\widehat{G}_{n x x}^{*}(\mathrm{y})-G_{x}(y)\right|=O\left(\tau_{n}\right)$, a.s., for each $y \in \Re$, and

(b) $n^{\frac{1}{2}} a_{\bar{n}}^{\frac{1}{2}}\left(\widehat{G}_{n x}^{*}(y)-G_{x}(y)\right) \longrightarrow N\left(0, \tau_{x}^{* 2}(y)\right)$, where $\sigma_{x}^{* 2}(y)=\sigma_{x}^{2}(y) / f(x)$ with $\sigma_{x}^{2}(y)$ as defined in Theorem $3.1\left(\frac{1}{b}\right)$.

REMARK 3.6. If $n a_{n}^{2 m+3} \rightarrow \theta \geq 0$, as $n \rightarrow \infty$, it can be shown, under the additional required conditions as in Corollary 3.1, that the asymptotic normality holds with bias term given by $b_{x}^{*}(y)=(\sqrt{\theta} /(m+1) !)\left\{\theta_{\bar{z} y^{* n+1}}^{*}\left[G_{x}(y)\right]+\left(\int t^{2} k_{1}(t) d t\right) \frac{\partial^{2}}{\partial x^{2}}\left[G_{x}(y)\right]\right\}$, where $\theta^{*}$ is as given by $(3.22)$. 


\section{Relative Defliciencies of $\widehat{G}_{n x}$ and $\widehat{G}_{n x}^{*}$}

We shall now investigate the conditions under which the "smoothed" c.e.d.f.'s $\widehat{G}_{m x}$ and $\widehat{G}_{n x}^{*}$ give better performance relative to the corresponding "unsmoothed" ones, namely, $G_{n x}$ and $G_{n \cdot x}^{*}$, respectively, given by

$$
G_{n x}(y)=\left(n a_{n}\right)^{-1}\left(d_{n}(x)\right)^{-1} \sum_{i=1}^{n} k_{1}\left(\left(F_{n}(x)-F_{n}\left(X_{i}\right) / a_{n}\right)\right) I_{[Y i \leq y]}, x<y<x ;
$$

and

$$
\left.G_{n x .}^{*}(y)=\left(n a_{n}\right)^{-1}\left(d_{n}^{*}(x)\right)^{-1} \sum_{i=1}^{n} k_{1}\left(\left(x-X_{i}\right) / a_{n}\right)\right) I_{\left[Y_{i \leq y]}\right.},-\infty<y<x ;
$$

where $d_{n}(x)=\left(n a_{n}\right)^{-1} \sum_{i=1}^{n} k_{1}\left(\left(F_{n}(x)-F_{n}\left(X_{i}\right)\right) / a_{n}\right)$ and $d_{n}^{*}(x)=\left(n a_{n}\right)^{-1} \sum_{i=1}^{n} k_{1}((x-$ $\left.\left.X_{i}\right) / a_{n}\right)$. Both $G_{n x}$ and $G_{n . x}^{*}$ have been extensively studied in literature (see Stute [16], [17]; see also Hardle et al.[4]).

We shall derive below relative deficiency expressions of $G_{n x .}\left(G_{n x .}^{*}\right)$ w.r. to $\widehat{G}_{n x x}\left(\widehat{G}_{n x .}^{*}\right)$ at a given point $y$ and show that, under certain conditions on the sequence $\left\{a_{n}\right\}$ of bandwidths and the kernel function employed, these relative deficiencies diverge to $x$, as $n \rightarrow x$. In above, our relative deficiency calculations are based on the mean square error (MSE) criterion. Also, for simplicity as well as for ready comparison of the present deficiency results with those of Falk [3] for the corresponding smoothed unconditional d.f. estimators, they are confined to the important special case when the bivariate kernel function used in $\widehat{G}_{n \cdot x}\left(\widehat{G}_{n t .}^{*}\right)$ is a "product" kernel function given by

$$
W_{n}(t, s)=k_{1}(t) K_{2}\left(s / a_{n}\right),-\infty<t, s<x,
$$

where $k_{1}$ is a symmetric kernel vanishing outside $[-1,1], K_{1}(t)=\int_{-x_{0}}^{t} k_{1}(u) d u$ and $K_{2}$ satisfies, for some $m \geq 1$,

$$
\begin{gathered}
K_{1}, K_{2} \in \mathscr{C}_{(m)}=\left\{L \in \mathscr{B}: \int d L=1, L(-x)=0, \int t^{p} d L(t)=0,1 \leq p \leq m\right. \text { and } \\
\left.\int|t|^{\mid n+1} d|L(t)|<x\right\},
\end{gathered}
$$

with $\mathscr{B}$ denoting the set of real valued functions of bounded variation. For $m \geq 1$, it is clear that the bivariate kernel function defined by (4.3) satisfies the assumptions A.III(i) to (v). We note, however, that if $m>1$, the function $k_{1}$ can not be a probability kernel and $K_{2}$ a probability distribution function, so that the estimator defined by (3.1) may not be a proper (empirical) distribution function. Despite this, in the interest of better overall efficiency and improved convergence of (kernel) estimators of density and distribution functions, the use of higher order kernels has been recommended by Bartlett [1] and subsequently by several other authors. The comparative results below, however, being valid for all $m \geq 1$, do cover the case $m=1$ when the smoothed c.e.d.f. $\widehat{G}_{n x . x}$, defined with $k_{1}$ a probability density and $K_{2}$ a proper d.f., satisfies the standard properties of a probability distribution function.

We shall now derive the large sample expressions, as $n \rightarrow \infty$, for the mean square 
errors of $\widehat{G}_{n x}(y)\left(\widehat{G}_{n x}^{*}(y)\right)$ and $G_{n x}(y)\left(G_{n x}^{*}(y)\right)$, respectively, the smoothed and unsmoothed RNN (NW) estimators of $G_{x}(y)$, for fixed $x \in \Lambda(F)$ and $y \in R$. These expressions are essential to our derivations of asymptotic relative efficiencies and deficiencies of these estimators.

First consider $\operatorname{MSE}\left(\widehat{G}_{n x}(y)\right)=E\left[\widehat{G}_{n x}(y)-G_{x}(y)\right]^{2}$. By setting $D_{n}(x)=1-t_{n}^{2}(x)$, we have from (3.2), for any fixed $0<\eta<1$,

$$
\begin{aligned}
\operatorname{MSE}\left[\left(\widehat{G}_{n x}(y)\right]\right. & =E\left[v_{n x}^{2}(y) / t_{n}^{2}(x)\right] \\
& =E\left\{v_{n x}^{2}(y)\left(1-D_{n}(x)\right)^{-1} I_{\left[\left|D_{n}(x)\right|<\eta\right]}\right\} \\
& \left.+E\left\{v_{n x}^{2}(y) / t_{n}^{2}(x)\right) I_{\left[\left|D_{n}(x)\right| \geq \eta\right]}\right\} \\
& =E\left[v_{n x}^{2}(y)\right]+E\left\{v_{n x}^{2}(y)\left[\left(1-D_{n}(x)\right)^{-1} I_{\left[\left|D_{n}(x)\right|<\eta\right]}-1\right]\right\} \\
& \left.+E\left\{v_{n x}^{2}(y) / t_{n}^{2}(x)\right) I_{\left[\left|D_{n}(x)\right| \geq \eta\right]}\right\} \\
& =E\left[v_{n x}^{2}(y)\right]+\varepsilon_{n x}^{(1)}(y)+\varepsilon_{n x x}^{(2)}(y) \text { (say) } \\
& =E\left[v_{n x}^{2}(y)\right]+o\left(n^{-1}\right), \text { as } n \rightarrow \infty
\end{aligned}
$$

provided we show that $\varepsilon_{n i x}^{(j)}(y)=o\left(n^{-1}\right)$ for $j=1,2$. This we shall demonstrate later (see $(4.40)$ and $(4.41))$. First we shall evaluate $E\left[v_{l t x}^{2}(y)\right]$ by studying the asymptotic expansion terms on the right of

$$
E\left[v_{n x}^{2}(y)\right]=\sum_{j=1}^{3} E\left[J_{n j}^{2}(y)\right]+2 \sum_{j \neq \mathbf{j}^{\prime}} E\left[J_{n j}(y) J_{n j^{\prime}}(y)\right] .
$$

For dealing $E\left[J_{n 1}^{2}(y)\right]$, note that by following arguments similar to those for (3.21) to (3.23); we obtain from (3.22) with $\varepsilon_{n}=c_{2}\left(n a_{n}\right)^{-1 / 2}$

$$
\begin{aligned}
E\left[J_{n 1}(y)-\bar{J}_{n 1}(y)\right]^{2} & \leq 4 \varepsilon_{n}^{2} k_{1}^{2}\left(t_{n}^{*}\right)\left[\int\left[K_{2}\left(\frac{y-v}{a_{n}}\right)-G_{x}(y)\right] d G_{x_{n}\left(t_{n}\right)}(v)\right]^{2} \\
& +c_{4} n^{-1} a_{n}^{-2}\left\{\left|F\left(x_{n}\left(1-\varepsilon_{n}\right)\right)-F\left(x_{n}(1)\right)\right|\right. \\
& \left.+\left|F\left(x_{n}(-1)\right)-F\left(x_{n}\left(\varepsilon_{n}-1\right)\right)\right|\right\} \\
& \leq c_{3}\left(n a_{n}\right)^{-1} k_{1}^{2}\left(t_{n}^{*}\right) a_{n}^{4}+c_{4}\left(n a_{n}^{2}\right)^{-1} \cdot a_{n}\left(n a_{n}\right)^{-1 / 2} \\
& =o(1 / n),
\end{aligned}
$$

as $n \rightarrow \infty$, since $k_{1}^{2}\left(t_{\mathrm{n}}^{*}\right) \rightarrow 0$, as $\left|t_{n}^{*}\right| \rightarrow 1$ and by assumption A. IV(ii), na $a_{n}^{3} \rightarrow \infty$. Thus

$$
\begin{aligned}
E\left[J_{n 1}^{2}(y)\right] & =E\left[\bar{J}_{n 1}^{2}\right]+o\left(n^{-1}\right) \\
& =E\left[\sum_{i=1}^{n} Z_{n i} / n\right]^{2}+o\left(n^{-1}\right) \\
& =n^{-1} E\left(Z_{n 1}^{2}\right)+n^{-1}(n-1)\left[E\left(Z_{n 1}\right)\right]^{2}+o\left(n^{-1}\right)
\end{aligned}
$$

with (cf. (3.13))

$$
\left.E\left(Z_{n 1}\right)=\int k_{1}(t)\left[\int K_{2}\left((y-v) / a_{n}\right)-G_{x}(y)\right] d G_{x_{n}(t)}(v)\right] d t
$$




$$
\begin{aligned}
& =\int k_{1}(t)\left[G_{x_{n}(t)}\left(y-a_{n} s\right) d K_{2}(s)-G_{x}(y)\right] d t \\
& =\int k_{1}(t)\left[G_{x_{n}(t)}(y)-G_{x}(y)+a_{n}^{m+1} \int s^{m+1} g_{x_{n}(t)}^{(m+1)}\left(y-\Delta_{n} a_{n} s\right) d K_{2}(s)\right] d t,
\end{aligned}
$$

where in (4.7), we have used integration by parts, Taylor's expansion and assumed that $K_{2} \in \mathfrak{t}_{(m)}$. Further, again assuming $K_{1} \in \mathscr{C}_{(m)}$ and using Taylor's expansion, we obtain in view of assumptions A.I and A.II that

$$
\int\left[G_{x_{n}(t)}(y)-G_{x}(y)\right] k_{1}(t) d t=O\left(a_{n}^{m+1}\right),
$$

as $n \rightarrow x ;(4.7)$ and (4.8) then yield

$$
E\left[Z_{n 1}\right]=O\left(a_{n}^{m+1}\right) .
$$

Also we can write for sufficiently large $n$, using Taylor's expansion and integration by parts,

$$
\begin{aligned}
& a_{n} E\left[Z_{n 1}^{2}\right]=\int k_{1}^{2}(t)\left\{\int\left[K_{2}\left((y-v) / a_{n}\right)-G_{x}(y)\right]^{2} d G_{x_{n}(t)}(v)\right\} d t \\
& =\int k_{1}^{2}(t)\left[\left.\left\{\mathrm{k}_{2}\left((y-v) / a_{n}\right)-G_{x}(y)\right\}^{2} G_{x_{n}(t)}(v)\right|_{-\infty} ^{\infty}-2 \int G_{x_{n}(t)}(v)\right. \\
& \left.\quad \times\left[K_{2}\left((y-v) / a_{n}\right)-G_{x}(y)\right] d K_{2}\left((y-v) / a_{n}\right)\right] d t \\
& =\int k_{1}^{2}(t)\left[G_{x}^{2}(y)+2 \int G_{x_{n}(t)}\left(y-a_{n} s\right) K_{2}(s) d K_{2}(s)\right. \\
& \left.\quad-2 G_{x}(y) \int G_{x_{n}(t)}\left(y-a_{n} s\right) d K_{2}(s)\right] d t \\
& =\int k_{1}^{2}(t)\left[G_{x}^{2}(y)+G_{x_{n}(t)}(y)-2 a_{n} g_{x_{n}(t)}(y) \int s K_{2}(s) d K_{2}(s)\right]+O\left(a_{n}^{m+1}\right) \\
& \left.\quad-2 G_{x}(y) G_{x_{n}(t)}(y)+O\left(a_{n}^{m+1}\right)\right] d t \\
& =\left(\int k_{1}^{2}(t) d t\right)\left[G_{x}(y)\left(1-G_{x}(y)\right)-2 a_{n} g_{x}(y) \int s K_{2}(s) d K_{2}(s)\right] \\
& \quad+O\left(a_{n}^{2}\right)+O\left(a_{n}^{m+1}\right),
\end{aligned}
$$

where in the last line we have used the symmetry of $k_{1}$ around zero $\left(\int t k_{1}^{2}(t) d t=0\right)$. From (4.6) to (4.10), for sufficiently large $n$ and under the conditions assumed above, we have

$$
\begin{aligned}
E\left[\bar{J}_{n 1}^{2}(y)\right]= & \left(n a_{n}\right)^{-1}\left(\int k_{1}^{2}(t) d t\right)\left[G_{x}(y)\left(1-G_{x}(y)\right)-2 a_{n} g_{x}(y) \int s K_{2}(s) d K_{2}(s)\right] \\
& +O\left(n^{-1} a_{n}\right)+O\left(a_{n}^{2 m+2}\right) \\
= & O\left(\left(n a_{n}\right)^{-1}\right) .
\end{aligned}
$$


Now for $J_{n 2},(4.3)$ yields $W_{n}^{(1,(0)}(t, s)=k_{1}^{(1)}(t) K_{2}\left(s / a_{n}\right)$ and $W_{n}^{*(1)}(t)=k_{1}^{(1)}(t)$ so that, defining $\bar{J}_{n 2}(y)$ analogously to $\bar{J}_{n 1}(y)$ (see $(3.2 \mathrm{a})$ ), we obtain from (3.2) that

$$
\begin{aligned}
\bar{J}_{n 2}^{2}(y)= & n^{-3} a_{n}^{-4}\left\{\sum _ { i = 1 } ^ { n } [ U _ { n } ( x ) - U _ { n } ( X _ { i } ) ] ^ { 2 } K _ { 1 } ^ { ( 1 ) ^ { 2 } } ( ( F ( x ) - F ( X _ { i } ) ) / a _ { n } ) \left[K_{2}\left(\left(y-Y_{i} / a_{n}\right)-G_{x}(y)\right]^{2}\right.\right. \\
& +\sum_{i \neq j}\left(U_{n}(x)-U_{n}\left(X_{i}\right)\right)\left(U_{n}(x)-U_{n}\left(X_{j}\right)\right) k_{1}^{(1)}\left(\left(F(x)-F\left(X_{i}\right)\right) / a_{n}\right) \\
& \cdot k_{1}^{(1)}\left(\left(F(x)-F\left(X_{j}\right)\right) / a_{n}\right)\left[K_{2}\left(\left(y-Y_{i}\right) / a_{n}\right)-G_{x}(y)\right]\left[K_{2}\left(\left(y-Y_{j}\right) / a_{n}\right)-G_{x}(y)\right] \\
= & I_{1}+I_{2} \text { (say). }
\end{aligned}
$$

where for $I_{1}$, on taking expectation and using the fact that $\left(X_{i}, Y_{i}\right), i=1,2, \ldots, n$, are i.i.d. r.v.'s, we have

$$
\begin{gathered}
E\left(I_{1}\right)=n^{-2} a_{n}^{-4} E\left\{k_{1}^{(1)^{2}}\left(\left(F(x)-F\left(X_{1}\right)\right) / a_{n}\right) E\left(\left[U_{n}(x)-U_{n}\left(X_{1}\right)\right]^{2} \mid X_{1}\right)\right. \\
\left.\cdot E\left(\left[K_{2}\left(\left(y-Y_{1}\right) / a_{n}\right)-G_{x}(y)\right]^{2} \mid X_{1}\right)\right\},
\end{gathered}
$$

with

$$
\begin{aligned}
E\left(\left[U_{n}(x)-U_{n}\left(X_{1}\right)\right]^{2} \mid X_{1}\right)= & n^{-1} E\left\{\left[\sum_{i=2}^{n}\left(I_{\left[X_{i} \leq x\right]}-I_{\left[X_{i} \leq X_{1}\right]}-F(x)+F\left(X_{1}\right)\right)\right.\right. \\
& \left.\left.+\left(I_{\left\{X_{1}>x\right]}-F(x)+F\left(X_{1}\right)\right)\right]^{2} \mid X_{1}\right\} \\
\leq & \left|F(x)-F\left(X_{1}\right)\right|\left(1-\left|F(x)-F\left(X_{1}\right)\right|\right)+c_{1} n^{-1},
\end{aligned}
$$

for some constant $c_{1}>0$ and

$$
\begin{aligned}
E\left\{\left[K_{2}\left(\left(y-Y_{1}\right) / a_{n}\right)-G_{x}(y)\right]^{2} / X_{1}\right\} & \leq\left[G_{X_{1}}(y)\left(1-G_{x}(y)\right)-G_{x}(y)\left(G_{X_{1}}(y)-G_{x}(y)\right)\right. \\
& \left.-a_{n} g_{X_{1}}(y) \psi\left(K_{2}\right)+c_{2} a_{n}^{2}\right]
\end{aligned}
$$

for some constant $c_{2}>0$. From (4.13) to (4.15) on, setting $x_{n}(t)=F^{-1}\left(F(x)-a_{n} t\right)$ we obtain

$$
\begin{aligned}
\left|E\left(I_{1}\right)\right| \leq & \left|n^{-2} a_{n}^{-2} \int k_{1}^{(1)^{2}}(t)\right| t\left|\left(1-a_{n}|t|\right)\right| G_{x_{n}(t)}(y)\left(1-G_{x}(y)\right) \\
& -G_{x}(y)\left(G_{x_{n}(t)}(y)-G_{x}(y)\right)-a_{n} G_{x_{n}(t)}(y) \psi\left(K_{2}\right)+c_{2} a_{n}^{2}|d t| \\
& +O\left(\bar{n}^{-3} a_{n}^{-2}\right) \\
= & o\left(n^{-1} a_{n}\right),
\end{aligned}
$$

as $n \rightarrow \infty$, by the assumption A.IV(ii), since the integral on the right is $O(1)$, as $n \rightarrow \infty$. Similarly for $I_{n 2}$, noting that the expectations of expressoins under double summation do not depend on the pair $(i, j)$, we obtain from (4.12) that

$$
\begin{aligned}
E\left[I_{2}\right] & =n^{-2} a_{n}^{-4}(n-1) E\left\{k^{(1)}\left(\left(F(x)-F\left(X_{1}\right) / a_{n}\right)\right) k_{1}{ }^{(1)}\left(\left(F(x)-F\left(X_{2}\right)\right) / a_{n}\right)\right. \\
& \cdot E\left\{\left(K_{2}\left(\left(y-Y_{1}\right) / a_{n}\right)-G_{x}(y)\right) \mid X_{1}\right\} \cdot E\left\{\left(K_{2}\left(\left(y-Y_{2}\right) / a_{n}-G_{x}(y)\right) \mid X_{2}\right\}\right. \\
& \left.\cdot E\left\{\left(U_{n}(x)-U_{n}\left(X_{1}\right)\right)\left(U_{n}(x)-U_{n}\left(X_{2}\right)\right) \mid X_{1}, X_{2}\right\}\right\},
\end{aligned}
$$


where by reasoning similar to that for (4.14) and (4.15),

$$
\begin{aligned}
& E\left\{\left(U_{n}(x)-U_{n}\left(X_{1}\right)\right)\left(U_{n}(x)-U_{n}\left(X_{2}\right) \mid X_{1}, X_{2}\right\}\right. \\
& \quad=-\left[F(x)-F\left(X_{1}\right)\right]\left[F(x)-F\left(X_{2}\right)\right]+c_{3} n^{-1},
\end{aligned}
$$

for some constant $c_{3}>0$, and

$$
E\left\{K_{2}\left((y-Y) / a_{n}\right)-G_{x}(y) \mid X_{1}\right\}=\left(G_{X_{1}}(y)-G_{x}(y)\right)+c_{4} a_{n}^{2}
$$

for some constant $c_{u}>0$; from (4.17) to (4.19), thus, we obtain by employing again the transformation $u=x_{n}(t),-1 \leq t \leq 1$,

$$
\begin{aligned}
\left|E\left(I_{2}\right)\right| & \leq n^{-1} a^{-3}\left[\iint\left|k_{1}^{(1)}(t)\right|\left\{\left|F(x)-F\left(x_{n}(t)\right)\right|+c_{5} n^{-1}\right\}\right. \\
& \left.\cdot\left\{\left|G_{x_{n}(t)}(y)-G_{x}(y)\right|+c_{6} a_{n}^{2}\right\} d t\right]^{2} \\
& \leq c_{7} n^{-1} a_{n},
\end{aligned}
$$

for some positive constants $c_{5}, c_{6}$ and $c_{7}$, the last inequality following in view of the assumption A.I and A.II and the symmetry of $k_{1}$ around zero. From (4.12), (4.16), (4.20) and (4.35) below, we thus obtain

$$
E\left[J_{n 2}^{2}(y)\right]=O\left(n^{-1} a_{n}\right), \text { as } n \rightarrow x .
$$

As for $J_{n 3}^{2}$, we have from (3.2) that

$$
\begin{aligned}
E J_{n_{3}}^{2}(y) \leq & (1 / 4) n^{-4} a_{n}^{-6}\left\{\sum _ { i = 1 } ^ { n } E \left\{\left(U_{n}(x)-U_{n}\left(X_{\mathrm{i}}\right)\right)^{4}\left(k_{1}^{(2)}\left(\Delta_{i n}\right)\right)^{2}\right.\right. \\
& \left.\cdot\left[K_{2}\left(\left(y-Y_{i}\right) / a_{n}\right)-G_{x}(y)\right]^{2}\right\} \\
+ & \sum_{i \neq j} E\left\{\left(U_{n}(x)-U_{n}\left(X_{i}\right)\right)^{2}\left(U_{n}(x)-U_{n}\left(X_{j}\right)\right)^{2} k_{1}^{(2)}\left(\Delta_{i n}\right) k_{1}^{(2)}\left(\Delta_{j n}\right)\right. \\
\cdot & {\left[K_{2}\left(\left(y-Y_{i}\right) / a_{n}\right)-G_{x}(y)\right] } \\
& {\left.\left[K_{2}\left(\left(y-Y_{i}\right) / a_{n}\right)-G_{x}(y)\right]\right\} . } \\
= & I_{3}+I_{4} \text { (say), }
\end{aligned}
$$

where for $I_{3}$ and $I_{4}$, using the boundedness of $k_{1}^{(2)},(4.15),(4.19)$ and arguments similar to those for (4.14) and (4.18), we obtain

$$
\begin{aligned}
\left|I_{3}\right| & \leq c_{8} n^{-4} a_{n}^{-6} n a_{n} \int_{-1}^{1}\left|F(x)-F\left(x_{n}(t)\right)\right|^{2} d t \\
& \leq c_{9} n^{-3} a_{n}^{-3}=o\left(n^{-2}\right)
\end{aligned}
$$

and

$$
\begin{aligned}
\left|I_{4}\right| & \leq c_{10} n^{-2} a_{n}^{-4}\left(\int k_{1}^{(2)^{2}}\left(\Delta_{1 n}\right)\left[F(x)-F\left(x_{n}(t)\right)\right]^{2} d t\right) \\
& =O\left(n^{-2} a_{n}^{-2}\right)=o\left(n^{-1} a_{n}\right)
\end{aligned}
$$


as $n \rightarrow \infty$. From (4.22) to (4.24), it follows that

$$
E\left[J_{n 3}^{2}(y)\right]=o\left(n^{-1} a_{n}\right), \text { as } n \rightarrow \infty .
$$

Now we deal with cross product terms: For this, first note that from (4.12), (4.21) and (4.25), it follows by Schwatz inequality and the assumption A.IV (ii) that

$$
\begin{aligned}
\left|E\left[J_{n 1}(y) J_{n 3}(y)\right]\right| & \leq\left[E J_{n 1}^{2}(y)\right]^{1 / 2} \cdot\left[E J_{n 3}^{2}(y)\right]^{1 / 2} \\
& =c_{11} n^{-1 / 2} a^{-1 / 2} \cdot n^{-1} a_{n}^{-1} \\
& =o\left(n^{-1}\right)
\end{aligned}
$$

and similarly

$$
\begin{aligned}
\left|E\left[J_{n 2}(y) J_{n 3}(y)\right]\right| & \leq c_{12} n^{-1 / 2} a_{n}^{1 / 2} \cdot n^{-1} a_{n}^{-1} \\
& =o\left(n^{-1} a_{n}\right)
\end{aligned}
$$

and further that

$$
\begin{aligned}
E\left[\bar{J}_{n 1}(y) \bar{J}_{n 2}(y)\right]= & n^{-5 / 2} a_{n}^{-3}\left[n E\left\{\phi_{1}\left(X_{1}\right) \phi_{2}\left(X_{1}\right) E\left(\left[K_{2}\left(\left(y-Y_{1}\right) / a_{n}\right)-G_{x}(y)\right]^{2} \mid X_{1}\right)\right\}\right. \\
+ & n(n-1) E\left\{\phi_{1}\left(X_{1}\right) \phi_{2}\left(X_{2}\right) E\left(\left[K_{2}\left(\left(y-Y_{1}\right) / a_{n}\right)-G_{x}(y)\right] \mid X_{1}\right)\right. \\
& \left.\cdot E\left(\left[K_{2}\left(\left(y-Y_{2}\right) / a_{n}\right)-G_{x}(y)\right] \mid X_{2}\right)\right\} \\
= & I_{5}+I_{6} \text { (say) }
\end{aligned}
$$

where $\phi_{1}\left(X_{1}\right)=k_{1}^{(1)}\left(\left(F(x)-F\left(X_{1}\right)\right) / a_{n}\right)\left[U_{n}(x)-U_{n}\left(X_{1}\right)\right]$ and $\phi_{2}\left(X_{2}\right)=k_{1}((F(x)-$ $\left.\left.F\left(X_{2}\right)\right) / a_{n}\right)$. Noting that $E\left(\left[U_{n}(x)-U_{n}\left(X_{1}\right)\right] \mid X_{1}, X_{2}\right)=n^{-\frac{1}{2}}\left[I_{\left[X_{1} \leq x\right]}+I_{\left[X_{2} \leq x\right]}+I_{\left[X_{1} \leq X_{2}\right]}\right.$ $\left.-2 F(x)+2 F\left(X_{1}\right)\right]$ and $E\left(\left[U_{n}(x)-U_{n}\left(X_{1}\right)\right] \mid X_{1}\right)=n^{-\frac{1}{2}}\left[I_{\left[X_{1}>x\right]}-F(x)-F\left(X_{1}\right)\right]$, it follows from (4.15) and (4.19) that

$$
\begin{aligned}
\left|I_{5}\right| \leq c_{13} n^{-2} a_{n}^{-3} a_{n} \int\left|k_{1}^{(1)}(t)\right|\left|k_{1}(t)\right| d t & \\
& =O\left(n^{-2} a_{n}^{-2}\right)=o\left(n^{-1} a_{n}\right),
\end{aligned}
$$

and

$$
\begin{aligned}
\left|I_{6}\right| & \leq c_{14} n^{-1} a_{n}^{-3} a_{n}^{2} \iint \mid k_{1}^{(1)}\left(t_{1}\right)\left\|k_{1}\left(t_{2}\right)\right\| G_{x_{n}\left(t_{1}\right)}(y) \\
& -G_{x}(y)+c_{4} a_{n}^{2} \|\left(G_{x_{n}\left(t_{2}\right)}(y)-G_{x}(y)\right)+c_{4} a_{n}^{2} \mid d t_{1} d t_{2} \\
& =O\left(n^{-1} a_{n}\right)
\end{aligned}
$$

as $n \rightarrow \infty$, the last equality in (4.30) following as for (4.20). From (4.29) and (4.30), we obtain $\left|E\left(\bar{J}_{n 1}(y) \cdot \bar{J}_{n 2}(y)\right)\right|=O\left(n^{-1} a_{n}\right)$ which implies, in view of (4.5b), (4.11) and (4.21),

$$
\begin{aligned}
\left|E\left(J_{n 1}(y) J_{n 2}(y)\right)\right| \leq & \mid E\left(\left(\bar{J}_{n 1}(y) \bar{J}_{n 2}(y)\right) \mid\right. \\
& \left.+E\left|J_{n 1}(y)-\bar{J}_{n 1}(y)\right| \mid J_{n 2}(y)\right) \mid \\
& \left.+E\left|\bar{J}_{n 1}(y)\right| \mid J_{n 2}(y)-\bar{J}_{n 2}(y)\right) \mid \\
\leq & O\left(n^{-1} a_{n}\right)+o\left(n^{-1} a_{n}^{\frac{1}{2}}\right)
\end{aligned}
$$




$$
\begin{aligned}
& +n^{-\frac{1}{2}} a_{n^{-\frac{1}{2}}}\left(E\left[J_{n 2}(y)-\bar{J}_{n 2}\right]^{2}\right)^{1 / 2} \\
= & o\left(n^{-1}\right),
\end{aligned}
$$

provided we show that

$$
E\left[J_{n 2}(y)-\bar{J}_{n 2}(y)\right]^{2}=o\left(n^{-1} a_{n}\right)
$$

as $n \rightarrow x$. We now establish (4.32): From (3.2) and the steps for (3.22) and (4.5b), one obtains using boundedness of $k_{1}^{(1)}$ and $K_{2}$

$$
\begin{aligned}
\left|\bar{J}_{n 2}(y)-J_{n 2}(y)\right| \leq & c_{15} n^{-\frac{1}{2}} a_{n}^{-2} \iint_{\left[1-\varepsilon_{n} \leq|t| \leq 1\right]}\left|k_{1}^{(1)}(t)\right|\left|U_{n}(x)-U_{n}\left(x_{n}(t)\right)\right| \\
& \times d\left|H_{n}\left(x_{n}(t), v\right)-H\left(x_{n}(t), v\right)-H_{n}\left(x_{n}(1), v\right)+H\left(x_{n}(1), v\right)\right| \\
+ & c_{16} n^{-\frac{1}{2}} a_{n}^{-1} \int_{\left[1-\varepsilon_{n} \leq|t| \leq 1\right]}\left|k_{1}^{(1)}(t)\right|\left|U_{n}(x)-U_{n}\left(x_{n}(t)\right)\right| \\
& \times\left|\int\left[K_{2}\left((y-v) / a_{n}\right)-G_{x}(y)\right] d G_{x_{n}(t)}(v)\right| d t \\
\leq & c_{17} n^{-1} a_{n}^{-2} \int_{\left[1-\varepsilon_{n} \leq|t| \leq 1\right]}\left|U_{n}(x)-U_{n}\left(x_{n}(t)\right)\right| d\left|U_{n}\left(x_{n}(t)\right)-U_{n}\left(x_{n}(1)\right)\right| \\
+ & c_{18} n^{-\frac{1}{2}} a_{n}^{-1} \varepsilon_{n}\left|k_{1}^{(1)}\left(t_{n}^{*}\right)\right|\left|U_{n}(x)-U_{n}\left(x_{n}\left(t_{n}^{*}\right)\right)\right| \\
& \times\left|\int\left[K_{2}\left((y-v) / a_{n}\right)-G_{x}(y)\right] d G_{x\left(t_{n}^{\prime}\right)}(v)\right|
\end{aligned}
$$

where $1-\varepsilon_{n} \leq\left|t_{n}^{*}\right| \leq 1$ and $\varepsilon_{n}=c\left(n a_{n}\right)^{-\frac{1}{2}}$. Now squaring and taking expectation on both sides, we obtain

$$
\begin{aligned}
E\left[\bar{J}_{n 2}(y)-J_{n 2}(y)\right]^{2} & \leq c_{19} n^{-2} a_{n}^{-4}\left\{E\left|U_{n}\left(x_{n}\left(1-\varepsilon_{n}\right)\right)-U_{n}\left(x_{n}(1)\right)\right|^{4}\right. \\
& \left.+E\left|U_{n}\left(x_{n}(-1)\right)-U_{n}\left(x_{n}\left(\varepsilon_{n}-1\right)\right)\right|^{4}\right\} \\
& +E\left[U_{n}(x)-U_{n}\left(x_{n}\right)(1)\right]^{2}\left[U_{n}\left(x_{n}\left(1-\varepsilon_{n}\right)\right)-U_{n}\left(x_{n}(1)\right)\right]^{2} \\
& +E\left[U_{n}(x)-U_{n}\left(x_{n}(1)\right]^{2}\left[U_{n}\left(x_{n}(-1)\right)-U_{n}\left(x_{n}\left(\varepsilon_{n}-1\right)\right)\right]^{2}\right. \\
& +c_{20} n^{-1} a_{n}^{-2} \varepsilon_{n}^{2} k_{1}^{(1)^{2}}\left(t_{n}^{*}\right) E\left[U_{n}(x)-U_{n}\left(x_{n}\left(t_{n}^{*}\right)\right)\right]^{2}
\end{aligned}
$$

Following the arguments used for (4.22) to (4.24), we obtain

$$
\begin{aligned}
E\left[\bar{J}_{n 2}(y)-J_{n 2}(y)\right]^{2} & \leq c_{21} n^{-2} a_{n}^{-4}\left[a_{n}^{2} \varepsilon_{n}^{2}+a_{n}^{2} \varepsilon_{n}\right]+c_{22} n^{-1} a_{n}^{-2} \varepsilon_{n}^{2} a_{n} \\
& =c_{21} n^{-1} a_{n}\left[n^{-2} a_{n}^{-4}+n^{-3 / 2} a_{n}^{-7 / 2}\right]+c_{22} n^{-1} a_{n}\left(n^{-1} a_{n}^{-3}\right) \\
& =o\left(n^{-1} a_{n}\right)
\end{aligned}
$$

as $n \rightarrow x$. This proves (4.32). Thus from (4.5a), (4.6), (4.11), (4.21), (4.25) to (4.27) and (4.31), we obtain that

$$
E\left[v_{n x}^{2}(y)\right]=E\left[J_{n 1}^{2}(y)\right]+o(1 / n),
$$

as $n \rightarrow \infty$. 
We now turn to $\varepsilon_{n t x}^{(i)}(y), i=1,2$ defined in (4.5). First consider $\varepsilon_{l l x}^{(1)}(y)$ : Now on the set $\left\{\left|D_{n}(x)\right|=\left|1-t_{n}^{2}(x)\right|<\eta<1\right\}$ expanding $\left[1-D_{n}(x)\right]^{-1}$ in powers of $D_{n}=D_{n}(x)$, we obtain

$$
\begin{aligned}
\left|\varepsilon_{n x}^{(1)}(y)\right| & =\left|-E\left(v_{n x x}^{2}(y) I_{\left[\left|D_{n}\right| \geq \eta\right]}\right)+E\left(v_{n x .}^{2}(y) I_{\left[\left|D_{n}\right|<n\right]}\left(D_{n}+D_{n}^{2}+\ldots\right)\right)\right| \\
& \leqslant\left[E v_{n x x}^{4}(y)\right]^{1 / 2}\left(P\left[\left|D_{n}\right| \geq \eta\right]\right)^{1 / 2} \\
& +(1-\eta)^{-1}\left[E v_{n x}^{4}(y)\right]^{1 / 2}\left(E\left(D_{n}^{2}\right)\right)^{1 / 2} \\
& =\left[E v_{n x x}^{4}(y)\right]^{1 / 2} \cdot\left(E D_{n}^{2}\right)^{1 / 2}\left[1+(1-\eta)^{-1}\right] .
\end{aligned}
$$

Now after some lengthy computations one can show that

$$
\begin{aligned}
E\left[v_{n . x}^{4}(y)\right] & =c\left(n a_{n}\right)^{-2}\left(\int k_{1}^{2}(u) d u\right)^{2}\left\{G_{x}(y)\left(1-G_{x}(y)\right)-2 a_{n} g_{x}(y) \psi\left(K_{2}\right)\right\}^{2} \\
& +o\left(n^{-2} a_{n}^{-1}\right)
\end{aligned}
$$

and by using Taylor's expansion for $t_{n}(x)$ as for $v_{n x}(y)$ and arguments used for evaluating $E\left(J_{n j}^{2}(y)\right), E\left(J_{n j}^{4}(y)\right)$, it can be shown after extensive computations that

$$
\begin{aligned}
E\left[D_{n}^{2}\right] & =E\left[1-t_{n}^{2}(x)\right]^{2} \\
& =1-2 E\left[t_{n}^{2}(x)\right]+E\left[t_{n}^{4}(x)\right] \\
& =c\left(n a_{n}\right)^{-1}\left(\int k_{1}^{2}(t) d t\right)+o\left(n^{-1}\right),
\end{aligned}
$$

as $n \rightarrow x$. Using (4.38) and (4.39) in (4.37), we obtain

$$
\begin{aligned}
\left|\varepsilon_{n x}^{(1)}(y)\right| & =O\left(\left(n a_{n}\right)^{-1}\left(n a_{n}\right)^{-1 / 2}\right) \\
& =o\left(n^{-1}\right)
\end{aligned}
$$

as $n \rightarrow x$. To prove a similar result for $\left|\varepsilon_{n, x}^{(2)}(y)\right|$, we need the order of the fourth moment of $D_{n}$, viz., (obtainable after extensive computations)

$$
\left.E\left[D_{n}^{4}\right]=c\left(n a_{n}\right)^{-2}\left(\int k_{1}^{2}(t) d t\right)\right)^{2}+o\left(n^{-2} a_{n}^{-1}\right)
$$

as $n \rightarrow \infty$. For the case $m=1$, since $\left|\widehat{G}_{n x x}(y)-G_{x}(y)\right|=\left|v_{n x}(y) / t_{n}(x)\right|$ is bounded, we obtain using (4.41) that

$$
\begin{aligned}
\left|\varepsilon_{n x}^{(2)}(y)\right| & \leq c P\left[\left|D_{n}\right| \geq \eta\right] \\
& \leq c \eta^{-1} E\left[D_{n}^{4}\right] \\
& =o\left(n^{-1}\right) .
\end{aligned}
$$

For the general case $m>1$, we need to impose a condition on the estimator, viz.,

$$
\sup _{n} E\left|\widehat{G}_{n x}(y)\right|^{2 p}<x \text { for some } p \geq 4
$$

then taking $p=4$, and using Holder's inequality, we obtain

$$
\left|\varepsilon_{n x}^{(2)}(y)\right| \leq\left[E\left(\widehat{G}_{n x}^{8}(y)+1\right)\right]^{1 / 4}\left(P\left[\left|D_{n}\right| \geq \eta\right]\right)^{\frac{3}{4}}
$$




$$
\begin{aligned}
& \leq c\left(E\left[D_{n}^{4}\right]\right)^{\frac{3}{4}} \\
& =o\left(n^{-1}\right),
\end{aligned}
$$

as $n \rightarrow \infty$, where for the last inequality we have utilized the order of $E\left[D_{n}^{4}\right]$ given by (4.4). Thus (4.40) and (4.44) establish (4.5).

From (4.5), (4.11) and (4.36), we obtain as $n \rightarrow \infty$

$$
\begin{aligned}
\operatorname{MSE}\left(\widehat{G}_{n x}(y)\right)= & \left(n a_{n}\right)^{-1}\left(\int k_{1}^{2}(t) d t\right)\left[G_{x}(y)\left(1-G_{x}(y)\right)\right. \\
& \left.-2 a_{n} g_{x}(y) \int s K_{2}(s) d K_{2}(s)+o\left(a_{n}\right)\right] \\
& +O\left(a_{n}^{2 m+2}\right) \\
= & \left(n a_{n}\right)^{-1}\left(\int k_{1}^{2}(t) d t\right)\left[G_{x}(y)\left(1-G_{x}(y)\right)\right. \\
& \left.-2 a_{n} g_{x}(y) \int s K_{2}(s) d K_{2}(s)+o\left(a_{n}\right)\right],
\end{aligned}
$$

provided we assume that $n a_{n}^{5} \rightarrow 0$, as $n \rightarrow \infty$ for the case $m=1$; for the general case $m$ $>1$, the result (4.45) holds if we assume $n a_{n}^{2 m+3} \rightarrow 0$, as $n \rightarrow \infty$, and that the condition (4.43) holds.

By following similar arguments, under the same conditions (excepting the not relevant ones on $K_{2}$ ) we obtain for the unsmoothed conditional empirical distribution function $G_{n x}(y)$ that, as $n \rightarrow \infty$,

$$
\operatorname{MSE}\left(G_{n x}(y)\right)=\left(n a_{n}\right)^{-1}\left(\int k_{1}^{2}(t) d t\right)\left[G_{x}(y)\left(1-G_{x}(y)\right)+o\left(a_{n}\right)\right] .
$$

We thus have

Lemma 4.1. Suppose that the assumption A.I(ii), A.II hold and the kernel functions $K_{1}, K_{2}$ defining $W_{n}(t, s)$ in (4.3) satisfy (4.4) for some $m \geq 1$ with (4.43) also holding if $m>1$. Further, assume for the bandwidth sequence $\left\{a_{n}\right\}$ that, as $n \rightarrow \infty$, in addition to A.IV(i) and (ii), $n a^{2 m+3} \rightarrow 0$, as $n \rightarrow \infty$. Then for sufficiently large $n$, the $\operatorname{MSE}\left(\widehat{G}_{n . x}(y)\right)$ and $\operatorname{MSE}\left(G_{n, x}(y)\right)$, for fixed $x \in \Lambda(F)$ and $y \in \mathscr{R}$ with $W_{n}(t, s)$, as given in (4.3), are given by (4.45) and (4.46) respectively.

Proof. The proof has been accomplished as assertions (4.45) and (4.46) above.

We now derive the (asymptotic) expressions, as $n \rightarrow \infty$, for the MSE's of the NW type smoothed and unsmoothed estimators $\widehat{G}_{n x x}^{*}(y)$ and $G_{n x}^{*}(y)$ of the conditional d.f. $G_{x}(y)$, for fixed $x \in \Lambda(F)$ and $y \in \mathscr{R}$, respectively, given by

$$
\widehat{G}_{n x}^{*}(y)=\left(n a_{n}\right)^{-1}\left(d_{n}^{*}(x)\right)^{-1} \sum_{i=1}^{n} k_{1}\left(\left(x-X_{i}\right) / a_{n}\right) K_{2}\left(\left(y-Y_{i}\right) / a_{n}\right)
$$

and 


$$
G_{n x}^{*}(y)=\left(n a_{n}\right)^{-1}\left(d_{n}^{*}(x)\right)^{-1} \sum_{i=1}^{n} k_{1}\left(\left(x-X_{i}\right) / a_{n}\right) I_{[Y i \leq y]},
$$

where $d_{n}^{*}(x)=\left(n a_{n}\right)^{-1} \sum_{i=1}^{n} k_{1}\left(\left(x-X_{i}\right) / a_{n}\right)$. By noting that, as $n \rightarrow x, d_{n}^{*}(x) \rightarrow f(x)$ with probability one and arguing as for (4.5) and (4.6), we have for sufficiently large $n$

$$
\begin{aligned}
E\left[G_{n x}^{*}(y)-G_{x}(y)\right]^{2} & \approx f^{-2}(x)\left[\operatorname{Var}\left(J_{n x}^{*}(y)\right)+\left(\mu_{n x x}^{*}(y)\right)^{2}\right] \\
& =f^{-2}(x)\left[n^{-1} E\left(Z_{n 1}^{* 2}\right)+n^{-1}(n-1)\left(E\left(Z_{n 1}^{*}\right)\right)^{2}\right],
\end{aligned}
$$

where $J_{n 1}^{*}(y)=n^{-1} \sum_{i=1}^{N} Z_{n i}^{*}, \mu_{n}^{*}(y)=E\left[J_{n 1}^{*}(y)\right]$ and

$$
\mathrm{Z}_{n i}^{*}=a_{n}^{-1} k_{1}\left(\left(x-X_{i}\right) / a_{n}\right)\left[K_{2}\left(\left(y-Y_{i}\right) / a_{n}\right)-G_{x}(y)\right],
$$

$i=1,2, \ldots n$. Now using below the transformation $a_{n} t=x-u$ and $a_{n} s=y-v$, and integration by parts, we have for sufficiently large $n$

$$
\begin{aligned}
E\left[Z_{n 1}^{*}\right] & =a_{n}^{-1} \int k_{1}\left((x-u) / a_{n}\right)\left[\int\left(K_{2}\left((y-v) / a_{n}\right)-G_{x}(y)\right) d G_{u}(v)\right] d F(u) \\
& =\int k_{1}(t)\left[\int G_{\left(x-a_{n} t\right)}\left(y-a_{n} s\right) d K_{2}(s)-G_{x}(y)\right] f\left(x-a_{n} t\right) d t \\
& =O\left(a_{n}^{m+1}\right)
\end{aligned}
$$

In concluding the last assertion in (4.49), we have specifically used the fact that $K_{1}, K_{2}$ $\in \mathscr{C}_{(m)}$. Further similarly as in (4.10), for sufficiently large $n$, we have using (4.26)

$$
\begin{aligned}
a_{n} E\left[Z_{n 1}^{* 2}\right]= & \int k_{1}^{2}(t)\left[\int\left[K_{2}\left((y-v) / a_{n}\right)-G_{x}(y)\right]^{2} d G_{\left(x-a_{n} t\right)}(v)\right] f\left(x-a_{n} t\right) d t \\
= & \int k_{1}^{2}(t)\left[G_{x}^{2}(y)+2 \int G_{\left(x-a_{n} t\right)}\left(y-a_{n} s\right) K_{2}(s) d K_{2}(s)\right. \\
& \left.-2 G_{x}(y) \int G_{x-a_{n} t}\left(y-a_{n} s\right) d K_{2}(s)\right] f\left(x-a_{n} t\right) d t \\
= & f(x)\left(\int k_{1}^{2}(t) d t\right)\left[G_{x}(y)\left(1-G_{x}(y)\right)\right. \\
& -2 a_{n} g_{x}(y) \int s K_{2}(s) d K_{2}(s)+O\left(a_{n}^{2}\right)+O\left(a_{n}^{m+1}\right) .
\end{aligned}
$$

Thus from (4.47), (4.49) and (4.51), we obtain

$$
\begin{aligned}
\operatorname{MSE}\left(\bar{G}_{n x}^{*}(y)\right)= & (f(x))^{-1}\left(n a_{n}\right)^{-1}\left(\int k_{1}^{2}(t) d t\right)\left[G_{x}(y)\left(1-G_{x}(y)\right)\right. \\
& \left.-2 a_{n} g_{x}(y) \int s K_{2}(s) d K_{2}(s)+O\left(a_{n}^{m+1}\right)\right]+O\left(a_{n}^{2 m+2}\right) \\
= & f^{-1}(x)\left(n a_{n}\right)^{-1}\left(\int k_{1}^{2}(t) d t\right)\left[G_{x}(y)\left[1-G_{x}(y)\right)\right. \\
& \left.-2 a_{n} g_{x}(y) \int s K_{2}(s) d K_{2}(s)+o\left(a_{n}\right)\right]
\end{aligned}
$$


for sufficiently large $n$, provided we assume that $n a_{n}^{2 m+3} \rightarrow 0$, as $n \rightarrow \infty$.

By following similar arguments and under the same assumptions as for (4.52) (except for those on $K_{2}$ ), we obtain for the unsmoothed $N W$-type conditional empirical d.f. $G_{n x}^{*}(y)$, that as $n \rightarrow x$

We can thus state

$$
\operatorname{MSE}\left(G_{n x}^{*}(y)\right)=f^{-1}(x)\left(n a_{n}\right)^{-1}\left(\int k_{1}^{2}(t) d t\right)\left[G_{x}(y)\left(1-G_{x}(y)+o\left(a_{n}\right)\right] .\right.
$$

Lemma 4.2. Suppose that assumption A.I(ii), (iii), A.II hold and kernels $K_{1}$ and $K_{2}$ defining $W_{n}(t, s)$, belong to $G_{(m)}$ for an $m \geq 1$ with (4.43) also holding if $m>1$. Further assume that the bandwidth sequence $\left\{a_{n}\right\}$ satisfies $a_{n} \rightarrow 0, n a_{n} \rightarrow \infty$, but na $a_{n}^{2 m+3}$ $\rightarrow 0$, as $n \rightarrow x$. Then the $\operatorname{MSE}\left(\widehat{G}_{n x}^{*}(y)\right)$ and $\operatorname{MSE}\left(G_{n x}^{*}(y)\right)$, for fixed $x \in \Lambda(F)$ and $y \in \Re$ and $W_{n}(t, s)$, defined by (4.3), are given, respectively, by (4.52) and (4.53) for sufficiently large $n$.

Remark 4.1. Bandwidth Selection. Combining arguments of Corollary 3.1 with equation (4.45), one can rewrite (4.45) more precisely as

$$
\begin{aligned}
\operatorname{MSE}\left(G_{n . x}(y)\right)= & n^{-1} a_{n}^{-1}\left(\int k_{1}^{2}(t) d t\right) G_{x}(y)\left(1-G_{x}(y)\right) \\
& -n^{-1}\left(\int k_{1}^{2}(t) d t\right) 2 g_{x}(y) \int s K_{2}(s) d K_{2}(s) \\
& +a_{n}^{2 m+2} \bar{b}_{x}^{2}(y)+o\left(n^{-1} a_{n}\right),
\end{aligned}
$$

where $\bar{b}_{x}(y)$ is $b_{x}(y)$ defined in Corollary 3.1 with $\theta$ and $\theta^{*}$ replaced, respectively, by 1 and $\int s^{m+1} d K_{2}(s)$. This equation yields the asymptotically "optimal" bandwidth minimizing $\operatorname{MSE}\left(G_{n x}(y)\right)$ as $a_{n, o p t}=\alpha\left(k_{1}, K_{2}, G\right) n^{-1 /(2 m+3)}$ with $\alpha\left(k_{1}, K_{2}, G\right)=$ $\left[\left(\int k_{1}^{2}(t) d t\right) G_{x}(y)\left(1-G_{x}(y)\right) / 2(m+1) \bar{b}_{x}^{2}(y)\right]^{1 /(2 m+3)}$. Thus the "optimal" bandwidth is of order $n^{-1 / 5}$ for $m=1$ and $n^{-1 /(2 m+3)}$ in general when $m>1$. In practice, one may use a bandwidth of slightly higher order than the "optimal" one, especially while choosing suitably the same band width for all values of $y$. Alternatively, for the "optimal" bandwidth one would need to replace $G_{x}(y)$ and its required partial derivatives in $\bar{b}_{x}(y)$ by their respective preliminary estimates. This remark applies, with appropriate modifications, to bandwidth selection in case of $N W$ estimator $G_{n x}^{*}$ as well.

\section{Relative Efficiency and Deficiency}

From Lemmas 4.1 and 4.2, it follows that asymptotically, as $n \rightarrow \infty$, the smoothed and unsmoothed RNN (NW) type (appropriately normalized) kernel estimators $G_{n x}(y)\left(G_{n x}^{*}(y)\right)$ and $G_{n x}(y)\left(G_{n x}^{*}(y)\right)$ of $G_{x}(y)$, for fixed $x \in \Lambda(F)$ and $y \in \mathscr{R}$ have asymptotically the same mean, variance or MSE. For comparing the performance of the above smoothed vs. unsmoothed estimators, thus, it is necessary to invoke a higher order efficiency, namely, that based on the concept of Relative Deficiency introduced by Hodges and Lehmann. For this, first note from the expressions defining 
$G_{n x}(y)$ or $G_{n x}(y)$, that the probability that an observation $\left(X_{i}, Y_{i}\right)$ will play an "effective" role in the definition, say, in the RNN case is $P\left[\left|F(x)-F\left(X_{i}\right)\right| \leq a_{n} \mid=P\left[F\left(X_{i}\right) \in\right.\right.$ $\left.\left(F(x)-a_{n}, F(x)+a_{n}\right)\right]=2 a_{n}$ (and in the NW case $P\left[X_{i} \in\left(x-a_{n}, x+a_{n}\right)\right]=F(x+$ $\left.\left.a_{n}\right)-F\left(x-a_{n}\right) \approx 2 a_{n} f(x)\right)$. Consequently the "effective" sample size in the above definitions is of the order $n a_{n}$, as $n \rightarrow x$.

Accordingly for the definition of Relative Efficiency and Deficiency, we set $N(n)=$ $\left[n a_{n}\right]$ and denote by

$$
\mathcal{N}(n)=\min \left\{R=\left[r a_{r}\right]: \operatorname{MSE}\left(G_{r x}(y)\right) \leq M S E G_{n x}(y)\right\} ;
$$

then $[N(n) / N(n)]$ and $[N(n)-N(n)]$ are called the Relative Efficiency and (relative) Deficiency, respectively, and their limiting values $A R E\left(G_{n x}(y), \widehat{G}_{n x}(y)\right)=\lim _{n \rightarrow \infty}[\mathcal{N}(n) /$ $N(n)]$ and $A R D\left(G_{n x}(y), \widehat{G}_{n x}(y)=\lim _{n \rightarrow \infty}[\mathcal{N}(n)-N(n)]\right.$, if they exist, are called Asymptotic Relative Efficiency and Asymptotic Relative Deficiency, respectively, of $G_{n x}(x)$ w.r. to $\widehat{G}_{n x}(x)$. For the Nadaraya-Watson type smoothed and unsmoothed conditional empirical d.f.'s $\widehat{G}_{n . x}^{*}(y)$ and $G_{n x}^{*}(y)$, one may define $\mathcal{N}^{*}(n)$ as $\mathcal{N}(n)$ in $(4.54)$ by replacing in these $\widehat{G}_{n x}(y)$ and $G_{n x x}^{*}(y)$ with $\widehat{G}_{n x}(y)$ and $G_{n x}^{*}(y)$, respectively. The ARE and ARD of $G_{n x}^{*}(y)$ with respect to $\widehat{G}_{n x}^{*}(y)$ can then be defined similarly.

We can now state

Theorem 4.1. Assume that $\mathcal{N}(n) / n \rightarrow 1$ as $n \rightarrow x$. Then $($ a) Under the conditions of Lemma 4.1 and Lemma 4.2, respectively $(i) \operatorname{ARE}\left(G_{n x}(y), G_{n x}(y)\right)=\lim _{n \rightarrow x}[\hat{N}(n) /$ $N(n)]=1$ and $(i i) \quad A R E\left(G_{n x x}^{*}(y), G_{n x}^{*}(y)\right)=\lim _{n \rightarrow \infty}\left[N^{*}(n) / N(n)\right]=1$;

(b) Let $\psi\left(K_{2}\right)=2 \int s K_{2}(s) d K_{2}(s)$. Then under the conditions of Lemma 4.1 and Lemma 4.2 respectively, we have

(i) $\lim _{n \rightarrow \infty}\left(\frac{\mathcal{N}(n)-N(n)}{N(n) a_{n}}\right)=\frac{g_{x}(y) \psi\left(K_{2}\right)}{G_{x}(y)\left(1-G_{x}(y)\right)}$

and

(ii) $\lim _{n \rightarrow \infty}\left(\frac{N^{* *}(n)-N(n)}{N(n) a_{n}}\right)=\frac{g_{x}(y) \psi\left(K_{2}\right)}{G_{x}(y)\left(1-G_{x}(y)\right)}$

Proof. The proof of part (a) is straightforward and follows from (4.45), (4.46) and the definition of $\mathcal{N}(n)$ in (4.54):

$$
\lim _{n \rightarrow \infty}[N(n) / N(n)]=\lim _{n \rightarrow \infty} \frac{G_{x}(y)\left[1-G_{x}(y)\right]+o\left(a_{r(n)}\right)}{G_{x}(y)\left[1-G_{x}(y)\right]-a_{n} g_{x}(y) \psi\left(K_{2}\right)+o\left(a_{n}\right)}=1 .
$$

This completes the proof of part (a)(i). The proof of part (a)(ii) follows similarly from $(4.52),(4.53)$ and $(4.54)^{*}$, that is, (4.54) with $\mathcal{N}^{*}(n)$ in place of $\mathcal{N}(n)$.

For part (b)(i), from (4.55) we have

$$
\lim _{n \rightarrow \infty} \frac{\mathcal{N}(n)-N(n)}{N(n) a_{n}}=\lim _{n \rightarrow \infty} \frac{1}{a_{n}} \frac{a_{n g} g_{x}(y) \psi\left(K_{2}\right)+o\left(a_{r(n)}\right)+o\left(a_{n}\right)}{G_{x}(y)\left[1-G_{x}(y)\right]-a_{n} g_{x}(y) \psi\left(K_{2}\right)+o\left(a_{n}\right)}
$$




$$
=\frac{g_{x}(y) \psi\left(K_{2}\right)}{G_{x}(y)\left[1-G_{x}(y)\right]},
$$

where, to obtain last expression as limit, we have used the obvious result that $\left(a_{r(n)} / a_{n}\right)$ $\rightarrow 1$, as $n \rightarrow \infty$. The proof of part (b)(ii) follows similarly using (4.52), (4.53), (4.54) and the preceding arguments. The proof is complete.

CoRollary 4.1. If $\psi\left(K_{2}\right)>0$, then under the conditions of Lemma 4.1 (Lemma 4.2) the relative deficiency of $G_{n . x}(y)\left(G_{n x x}^{*}(y)\right)$ w.r. to $G_{n x}(y)\left(G_{n x}^{*}(y)\right)$ for fixed, $x \in \Lambda$ $(F)$ and $y \in \Re$ namely, $[\mathcal{N}(n)-N(n)] \rightarrow x\left(\left[\mathcal{N}^{*}(n)-N(n)\right] \rightarrow \infty\right)$, as $n \rightarrow \infty$.

Proof. Follows from Theorem 4.1.

REMARK 4.2. If we define Deficiency using the actual sample size ' $n$ ' instead of effective sample size ' $n a_{n}$ ', we still have similar results as seen below: Let $r(n)=$ $\min \left\{r: M S E\left(G_{r x}(y)\right) \leq M S E\left(\widehat{G}_{n x}(y)\right)\right\}$. We then have from (4.45) and (4.46)

$$
\frac{1}{r(n) a_{r(n)}}\left[\sigma_{x}^{2}(y)+o\left(a_{r(n)}\right)\right] \leq \frac{1}{n a_{n}}\left[\sigma_{x}^{2}(y)-a_{n} g_{x}(y) \psi\left(K_{2}\right)+o\left(a_{n}\right)\right],
$$

where $\sigma_{x}^{2}(y)=G_{x}(y)\left[1-G_{x}(y)\right]\left(\int k_{1}^{2}(t) d t\right)$. Since $r(n) \rightarrow x$ as $n \rightarrow x$, it is easily verified that, if $\psi\left(K_{2}\right)>0$, for sufficiency large $n$

$$
\begin{gathered}
\frac{r(n) a_{r(n)}}{n a_{n}} \geq 1, \text { and } \\
\frac{r(n)-n}{r(n) a_{r(n)}} \geq\left(\frac{1}{a_{r(n)}}-\frac{1}{a_{n}}\right)+\frac{g_{x}(y) \psi\left(K_{2}\right)}{\sigma_{x}^{2}(y)}+o\left(\frac{n}{r(n)}\right)+o(1) ;
\end{gathered}
$$

In fact, equality holds in (4.56) in the limit, as $n \rightarrow \infty$, irrespective of the sign of $\psi\left(K_{2}\right)$. If $\left\{a_{n}\right\}$ satisfies (for large $n$ )

$$
\frac{r(n) a_{r(n)}}{n a_{n}} \geq 1 \Leftrightarrow r(n) \geq n,
$$

then it is easy to see from (4.56) and (4.57) that

$$
\frac{r(n)-n}{n a_{n}} \geq \frac{g_{x}(y) \psi\left(K_{2}\right)}{\sigma_{x}^{2}(y)}+o(1)
$$

which implies that if $\psi\left(K_{2}\right)>0, r(n)-n \rightarrow \infty$ as $n \rightarrow \infty$ as in Corollary 4.1. The condition (4.58), in fact, implies that $\lim [r(n) / n]=1$ as $n \rightarrow \infty$. The usual choice $a_{n}=$ $n^{-\eta}$ with $(2 m+2)^{-1}<\eta<1 / 3$ satifies (4.58) in addition to other stipulated conditions as required in Theorem 4.1 .

Remark 4.3. From Corollary 4.1, it follows that if $K_{2}$ in (4.3) is chosen to satisfy the required conditions in (4.4) and that $\psi\left(K_{2}\right)=2 \int s K_{2}(s) d K_{2}(s)>0$, then since the asymptotic relative deficiencies $A R D\left(G_{n x}(y), \widehat{G}_{n \cdot x}(y)\right)$ and $A R D\left(G_{n x .}^{*}(y), \widehat{G}_{n x}(y)\right) \rightarrow \infty$, as $n \rightarrow \infty$, in either case of RNN or NW estimators above, smoothing with $K_{2}$ does bring about improvement in the performance of the above defined c.e.d.f.'s as estimators 
of $G_{x}(y)$, for all specified values of $x \in \Lambda(F)$ and $y \in \Re$. However, since $A R E\left(\widehat{G}_{n x}(y)\right.$, $\left.\widehat{G}_{n x}^{*}(y)\right)=f^{-1}(x)$ which in most situations is $\geq 1$, one should prefer the smoothed RNN type estimator over the corresponding NW type if both ARE and ARD are the criteria to be used in selecting the estimator.

\section{References}

[1] Bartlett, M.S.: Statistical estimation of density functions. Sankhyā, Ser. A. 25 (1963), $245-254$.

[2] Efron. B.: Bootstrap Methods: Another look at the jackknife, Ann. Statist. 7 (1979), 1-26.

[3] FALK, M.: Relative efficiency and deficiency of kernel type estimators of smooth distribution functions, Statistica Nerlandica 37 (1983), 73-83.

[4] Härdle, W., Jinssen, P. and Serfling, R.: Strong uniform convergence rates for estimators of conditional functions, Ann. Statist. 16 (1988), 1428-1449.

[5] Honvath, L. and Yandell, B.S.: Asymptotics of conditional empirical processes, J. Mult. Analysis 26 (1988), 184-206.

[6] Kiefer. J.: On large deviations of the empiric D.F. of vector chance variables and a law of iterated logarithm, Pacific J. Math. 11 (1961), 649-660.

[7] MACк, Y.P.: Remarks on some smoothed empirical distribution functions and processes, Bull. Inform. Cybernet. 21 (1984), 29-35

[8] Mehra K.L., Rama Krishnaiah, Y.S. and Rao, M. Sudhakara: Bahadur representation of sample conditional quantiles based on smoothed conditional empirical distribution function, Bull. Inform. Cybernet. 25 (1992), 99-107.

[9] Mehra, K.L., Rao, M. Sudhakara and Upadrasti, S.P.: A smooth conditional quantile estimator and related applications of the conditional empirical process, J. Mult. Analysis 37 (1991), 151-179.

[10] ReIss, R.D.: Nonparametric estimation of smooth distribution functions, Scan. J. Statist. 8, (1981). $116-119$.

[11] Samanta M.: Nonparametric estimation of conditional quantiles, Stat. \& Probab. Letters 7 (1989). 407-412.

[12] Serfling, R.J.: Approximation Theorems in Mathematical Statistics, John Wilcy \& Sons, 1980.

[13] Stone, C.J.: Consistent nonparametric regression, Ann. Statist. 5 (1977), 595-645.

[14] Stute, W.: The oscillation behavior of empirical processes, Ann. Probab. 10 (1982), 86-107.

[15] Stute, W.: Asymptotic normality of nearest neighbor regression function estimates, Ann. Statist. 12 (1984), 917-926.

[16] Stute W.: Conditional empirical processes, Ann. Statist, 14 (1986), 638-647.

[17] Stute W.: On almost sure convergence of conditional empirical distribution functions, Ann. Probab., 14 (1986), 891-901.

[18] Walter G. and Blum, J.: Probability density estimation using delta sequences, Ann. Statist. 7 (1979), $328-340$.

Received June 21, 1990

Revised June 11, 1991

Communicated by H. Yamato 